\title{
ANISOTROPIC MESH ADAPTATION FOR CRACK DETECTION IN BRITTLE MATERIALS
}

\author{
MARCO ARTINA*, MASSIMO FORNASIER* \\ STEFANO MICHELETTI ${ }^{\dagger}$, AND SIMONA PEROTTO ${ }^{\dagger}$
}

\begin{abstract}
The quasistatic brittle fracture model proposed by G. Francfort and J.-J. Marigo can be $\Gamma$-approximated at each time evolution step by the Ambrosio-Tortorelli functional. In this paper, we focus on a modification of this functional which includes additional constraints via penalty terms to enforce the irreversibility of the fracture as well as the applied displacement field. Secondly, we build on this variational model an adapted discretization to numerically compute the time-evolving minimizing solution. We present the derivation of a novel a posteriori error estimator driving the anisotropic adaptive procedure. The main properties of these automatically generated meshes are to be very fine and strongly anisotropic in a very thin neighborhood of the crack, but only far away from the crack tip, while they show a highly isotropic behavior in a neighborhood of the crack tip instead. As a consequence of these properties, the resulting discretizations follow very closely the propagation of the fracture, which is not significantly influenced by the discretization itself, delivering a physically sound prediction of the crack path, with a reasonable computational effort. In fact, we provide numerical tests which assess the balance between accuracy and complexity of the algorithm. We compare our results with isotropic mesh adaptation and we highlight the remarkable improvements both in terms of accuracy and computational cost with respect to simulations in the pertinent most recent literature.
\end{abstract}

1. Introduction and motivations. Mathematical models of the propagation of brittle fractures based on variational principles have arisen a strong interest in the past 15 years. On the one hand, they describe a mechanical problem which collects the interest of different disciplines, from physics of solids to mechanical engineering. On the other hand, they pose fascinating mathematical questions related to existence, regularity, and numerical simulation of physically sound solutions.

One of the most advocated models for quasistatic brittle fracture evolution was first presented by G. Francfort and J.-J. Marigo in [25]. This model is particularly relevant because it is able to predict complex crack paths, without making a priori assumptions on their possible propagation. The behavior of the fracture is defined by the minimization of the energy of the system following Griffith's principle of energy balance of two main terms: the first is the elastic energy and the second is a fictitious energy that represents the crack formation cost, typically assumed to be proportional to the surface of the created crack [29]. While in the original model of G. Francfort and J.-J. Marigo, the existence of the crack evolution is assumed, only with the works of G. Francfort and C. J. Larsen [26] and G. Dal Maso and R. Toader [20] the existence of a continuous time evolution of the quasistatic model was proved.

Despite the success of this model for its mathematical well-posedness and, at the same time, its rather general framework, mechanical engineers and physicists of solids tend to favor more realistic models, where a smoother process towards fracture is considered and a minimal cohesion between the surfaces of the crack is not negligible. From this perspective, the approximation made by L. Ambrosio and V. M. Tortorelli in [2] of the energy functional driving the quasistatic evolution of the Francfort-Marigo model is very interesting because the crack is identified by a smooth phase field

*Faculty of Mathematics, Technische Universität München, Boltzmannstrasse 3, 85748, Garching, Germany, Email: marco.artina@ma.tum.de, massimo.fornasier@ma.tum.de

${ }^{\dagger}$ MOX - Modeling and Scientific Computing, Department of Mathematics "F.Brioschi", Politecnico di Milano, Via Bonardi 9, I-20133 Milano, Italy, Email: stefano.micheletti@polimi.it, simona.perotto@polimi.it 
$v: \Omega \rightarrow[0,1]$, instead of a sharp lower dimensional set. For this reason, we consider exclusively a numerical analysis and simulation of a quasistatic evolution based on the Ambrosio-Tortorelli functional. Although it can be defined in any dimension and for any Lipschitz domain, for simplicity of presentation we assume that $\Omega \subset \mathbb{R}^{2}$ is a polygonal domain, by denoting $\mathbf{x}=\left(x_{1}, x_{2}\right)^{T}$ the generic point in $\Omega$.

Let us now introduce in more detail the model. We define a load which will drive the evolution of the crack. This function, $g:[0, T] \times \Omega \rightarrow \mathbb{R}$, is such that

$$
g(t)=\left\{\begin{array}{rl}
t & \text { on } \Omega_{D^{+}} \\
-t & \text { on } \Omega_{D^{-}} \\
0 & \text { elsewhere }
\end{array},\right.
$$

where $\Omega_{D^{ \pm}}=\Omega_{D^{+}} \cup \Omega_{D^{-}}$is the subdomain where the load is applied and the dependence on $\mathbf{x}$ is understood. We also define the space

$$
\mathcal{A}(g(t))=\left\{u \in H^{1}(\Omega):\left.u\right|_{\Omega_{D^{ \pm}}}=\left.g(t)\right|_{\Omega_{D^{ \pm}}}\right\}
$$

of the admissible solutions. We define the functional: $I_{\varepsilon}: H^{1}(\Omega) \times H^{1}(\Omega ;[0,1]) \rightarrow \mathbb{R}$ as

$$
I_{\varepsilon}(u, v)=\int_{\Omega}\left(v^{2}+\eta\right)|\nabla u|^{2} d \mathbf{x}+\kappa \int_{\Omega}\left[\frac{1}{4 \varepsilon}(1-v)^{2}+\varepsilon|\nabla v|^{2}\right] d \mathbf{x},
$$

where $0<\eta \ll \varepsilon \ll 1, \kappa>0, u \in \mathcal{A}(g(t))$, and $v \in H^{1}(\Omega ;[0,1])$. The first integral of (1.2) represents the elastic energy $E_{e}$ of the body, while the second one is the fictitious crack energy $E_{f}$. As proved in [9], this functional $\Gamma$-converges in $L^{1}(\Omega) \times L^{1}(\Omega)$ to the energy functional driving the evolution of the Francfort-Marigo model, as $\varepsilon \rightarrow 0$. This proof is built upon the original result of convergence made by L. Ambrosio and V.M. Tortorelli in [1] for the approximation of the Mumford-Shah functional [37]. Moreover, in [2], the proof of the existence of minimizers for (1.2) is provided for all $\varepsilon, \eta>0$. Alternative $\Gamma$-approximations results are addressed, e.g., in [5, 22].

Our numerical approximation will be based on a discretization in both space and time. Therefore, we introduce a time discretization $0=t_{0}<t_{1}<\ldots<t_{F}=T$. The evolution is driven by a process of minimization of (1.2) at each discrete time, which, for $t=t_{0}$, is given by

$$
\left(u_{\varepsilon}\left(t_{0}\right), v_{\varepsilon}\left(t_{0}\right)\right) \in \underset{\substack{u \in \mathcal{A}\left(g\left(t_{0}\right)\right), v \in H^{1}(\Omega ;[0,1])}}{\arg \min } I_{\varepsilon}(u, v),
$$

whereas, for subsequent times $t=t_{k}$, for $k=1, \ldots, F$, we seek a pair $\left(u_{\varepsilon}\left(t_{k}\right), v_{\varepsilon}\left(t_{k}\right)\right)$ such that

$$
\left(u_{\varepsilon}\left(t_{k}\right), v_{\varepsilon}\left(t_{k}\right)\right) \in \underset{\substack{u \in \mathcal{A}\left(g\left(t_{k}\right)\right), v \in H^{1}(\Omega ;[0,1]), v \leq v_{\varepsilon}\left(t_{k-1}\right)}}{\arg \min } I_{\varepsilon}(u, v) .
$$

The two components of the solution to (1.3) represent the displacement of the body and the phase field of the fracture. In particular, the fracture is identified by the subset of the domain where $v_{\varepsilon}\left(t_{k}\right)$ is close to zero. The transition layer between the two regions has a thickness of order $\varepsilon$ and the condition $v \leq v_{\varepsilon}\left(t_{k-1}\right)$ enforces the irreversibility of the crack [28]. 
While the existence of a continuous time evolution for the model evolving along global minimizers, as defined in (1.3), has been shown in [26, 20], it is more delicate to address the system development when it is evolving along local minimizers or critical points of the energy. In this paper, we actually consider a reliable numerical approximation of the evolution along critical points, as it would be more realistic from a physical point of view [19]. So far, accurate numerical simulations of the Francfort-Marigo model of fracture evolution are based on very fine ad hoc meshes, with an enormous computational effort $[9,6]$. After some attempts towards adaptive discretization of free-discontinuity functionals of Mumford-Shah type $[8,10,11,14$, 39], still we lack a reliable adaptive strategy which can successfully break the dilemma "the grid follows the fracture or the fracture follows the grid", with a proper balance between accuracy and complexity. In this paper, we make a yet another attempt to challenge this serious difficulty. We follow and combine both strategies suggested in the papers $[10,8]$; in particular, we merge the minimization of the AmbrosioTortorelli model with an adaptive anisotropic discretization by exploiting the fact that the considered model exhibits solutions with very steep features close to the crack. Moreover, as the crack evolves along a specific direction, the choice of an anisotropic adaptation can be really advantageous compared with a more standard isotropic approach. In more detail, the proposed adaptation procedure is driven by the residual associated with the gradient of the energy functional. To highlight the contribution of the present paper, we describe below its relevance with respect to the recent literature $[8,10]$.

The significant innovation with respect to [8], where adaptive anisotropic meshes are also considered, is twofold. On the one-hand, we deal with the Ambrosio-Tortorelli functional instead of the Mumford-Shah functional. On the other hand, while in [8] a heuristic Hessian-based approach is employed to drive the mesh adaptation, we resort to a metric-based procedure hinging on a sound error estimator.

The main improvements with respect to [10] are both in considering anisotropic meshes, in contrast to exclusively isotropic refinement, and the fact that we adapt the mesh at each minimization step. These apparently minor changes lead to considerable improvements both in terms of accuracy and computational cost. In particular, the proposed a posteriori error estimator has two main properties: the automatically generated meshes are very fine and strongly anisotropic in a thin neighborhood of the crack, whereas they show highly isotropic behavior in a neighborhood of the crack tip. As a consequence, the resulting discretization follows very closely the propagation of the fracture, which is not significantly influenced by the discretization, delivering a physically sound prediction of the crack path, with a reasonable computational effort.

The content of the paper is organized as follows: in Section 2, we introduce a mild modification of the Ambrosio-Tortorelli functional and we provide some results on its regularity. In Section 3, we introduce the anisotropic setting and we derive the anisotropic a posteriori error estimator. Then, two different adaptation algorithms are furnished in Section 4 and numerically validated on two test cases in Section 5.

2. The modified Ambrosio-Tortorelli model. The minimization process (1.3) requires minimizing a functional subject to constraints on both $u$ and $v$. In particular, we propose a minimization process where the constraints are relaxed through suitable penalty terms. This choice avoids us selecting special function spaces and allows us to pose the problem in $H^{1}(\Omega)$ for both $u$ and $v$. Moreover, it simplifies significantly the numerical implementation.

Before introducing the penalized functional, we properly rewrite the constraint on the 
function $v$. In fact, the condition

$$
v \leq v_{\varepsilon}\left(t_{k-1}\right)
$$

enforcing the irreversibility of the crack, cannot be easily implemented. So we follow an alternative criterion, first introduced by Bourdin in [6], where the irreversibility is provided by an equality constraint. In particular, if at time $t=t_{k-1}$ the set

$$
C R_{k-1}=\left\{\mathbf{x} \in \bar{\Omega} \mid v_{\varepsilon}\left(t_{k-1}\right)<\mathrm{CRTOL}\right\}
$$

is nonempty for a small specified tolerance CRTOL, the irreversibility is enforced by

$$
v_{\varepsilon}\left(\mathbf{x}, t_{i}\right)=0 \quad \forall \mathbf{x} \in C R_{k-1} \quad \text { and } \quad \forall i: k \leq i \leq F .
$$

Moving from this idea, we propose minimizing the following penalized functional

$$
\begin{aligned}
I_{\varepsilon, k}^{\text {penalty }}(u, v) & =\int_{\Omega}\left(v^{2}+\eta\right)|\nabla u|^{2} d \mathbf{x}+\kappa \int_{\Omega}\left[\frac{1}{4 \varepsilon}(1-v)^{2}+\varepsilon|\nabla v|^{2}\right] d \mathbf{x} \\
& +\frac{1}{\gamma_{A}} \int_{\Omega_{D^{ \pm}}}\left(g\left(t_{k}\right)-u\right)^{2} d \mathbf{x}+\frac{1}{\gamma_{B}} \int_{C R_{k-1}} v^{2} d \mathbf{x}
\end{aligned}
$$

where $\gamma_{A}$ and $\gamma_{B}$ are the two (small) penalty constants. Hence, the new optimization problem is

$$
\left(u_{\varepsilon}\left(t_{k}\right), v_{\varepsilon}\left(t_{k}\right)\right) \in \underset{\substack{u \in H^{1}(\Omega), v \in H^{1}(\Omega ;[0,1])}}{\arg \min } I_{\varepsilon, k}^{\text {penalty }}(u, v),
$$

for $k=1, \ldots, F$. Notice that, even though we are explicitly taking into account the constraint $v \in H^{1}(\Omega ;[0,1])$, hence assuming that $v$ takes exclusively values in $[0,1]$, shortly we shall prove that this boundedness is automatically fulfilled by the unconstrained minimization and one need not implement it in practice. Differently from the previous models $[6,10]$, we observe that for $t=t_{0}$, we can still use $(2.5)$ as a starting minimization, as the last penalization term vanishes. Since the constraints are clearly continuous, convex, and always non-negative, the proof of the convergence of the minimizers of (2.5) to ones fulfilling (2.3) instead of (2.1) in (1.3) for $\gamma_{A}, \gamma_{B} \rightarrow 0$, follows from the $\Gamma$-convergence theory [18].

From now on, we refer only to functional (2.4), and we simplify the notation by setting $\kappa=1$ and by adopting the short-hand notation

$$
\begin{aligned}
I(u, v) & =\int_{\Omega}\left[\left(v^{2}+\eta\right)|\nabla u|^{2} d \mathbf{x}+\alpha(1-v)^{2}+\varepsilon|\nabla v|^{2}\right] d \mathbf{x} \\
& +\frac{1}{\gamma_{A}} \int_{\Omega_{D^{ \pm}}}\left(g\left(t_{k}\right)-u\right)^{2} d \mathbf{x}+\frac{1}{\gamma_{B}} \int_{C R_{k-1}} v^{2} d \mathbf{x},
\end{aligned}
$$

with $\alpha=(4 \varepsilon)^{-1}$. Henceforth, we refer to (2.6) as to the modified Ambrosio-Tortorelli functional. Throughout the remaining part of this section, we mimic the analysis in Süli et al. [10] by suitably modifying it to deal with functional (2.6).

Using a truncation argument, it can be checked that any local minimizer $(u, v)$ of $I(\cdot, \cdot)$ in the $H^{1}(\Omega) \times H^{1}(\Omega)$ topology is such that $0 \leq v \leq 1$ a.e. in $\Omega$. Hence, we allow ourself to restrict the trial space for $v$ to $L^{\infty}(\Omega) \cap H^{1}(\Omega)$. 
Proposition 2.1. The functional $I(\cdot, \cdot)$ is Fréchet-differentiable in $H^{1}(\Omega) \times$ $\left(H^{1}(\Omega) \cap L^{\infty}(\Omega)\right)$.

Proof. The proof follows directly from Proposition 1.1 in [10]. In particular, the differentiability of the additional penalty terms is trivial and thus the penalty terms does not change the regularity of the functional $I(\cdot, \cdot)$.

Let us introduce now the Fréchet derivative of $I(w, z)$ in the direction $(\varphi, \psi)$, i.e.,

$$
\begin{aligned}
I^{\prime}(w, z ; \varphi, \psi)= & 2\left(\int_{\Omega}\left(z^{2}+\eta\right) \nabla w \cdot \nabla \varphi d \mathbf{x}+\frac{1}{\gamma_{A}} \int_{\Omega_{D^{ \pm}}}\left(w-g\left(t_{k}\right)\right) \varphi d \mathbf{x}\right) \\
+ & 2\left(\int_{\Omega}\left[z \psi|\nabla w|^{2}+\alpha(z-1) \psi+\varepsilon \nabla z \cdot \nabla \psi\right] d \mathbf{x}+\frac{1}{\gamma_{B}} \int_{C R_{k-1}} z \psi d \mathbf{x}\right) \\
=: & 2 a_{\gamma_{A}}(z ; w, \varphi)+2 b_{\gamma_{B}}(w ; z, \psi),
\end{aligned}
$$

where we have split the derivative in two parts; the first one, $a_{\gamma_{A}}$, associated with the derivative in the direction $\varphi$, and the second one, $b_{\gamma_{B}}$, related to the direction $\psi$. Accordingly, we define the notion of critical point for $I(\cdot, \cdot)$.

Definition 2.2. The pair $(u, v) \in H^{1}(\Omega) \times\left(H^{1}(\Omega) \cap L^{\infty}(\Omega)\right)$ is a critical point of $I(\cdot, \cdot)$ if $I^{\prime}(u, v ; \varphi, \psi)=0$ for all $\varphi \in H^{1}(\Omega)$ and for all $\psi \in\left(H^{1}(\Omega) \cap L^{\infty}(\Omega)\right)$. By the following proposition, we can get rid of the constraint on $v$, as anticipated above.

Proposition 2.3. If $(u, v) \in H^{1}(\Omega) \times\left(H^{1}(\Omega) \cap L^{\infty}(\Omega)\right)$ is a critical point of $I(\cdot, \cdot)$, then $0 \leq v(\mathbf{x}) \leq 1$ for a.e. $\mathbf{x} \in \Omega$.

Proof. Following the argument of Proposition 1.3 of [10], suppose that $(u, v)$ is a critical point of $I(\cdot, \cdot)$ and that $\Omega_{1}$ and $\Omega_{2}$ are the two subsets of $\Omega$ such that $\Omega_{1}=\{\mathbf{x} \in \Omega \mid v(\mathbf{x})>1\}, \Omega_{2}=\{\mathbf{x} \in \Omega \mid v(\mathbf{x})<0\}$, and $\left|\Omega_{1} \cup \Omega_{2}\right|>0$. Since $(u, v)$ is a critical point of $I(\cdot, \cdot)$, we have

$$
b_{\gamma_{B}}(u ; v, \psi)=0 \quad \forall \psi \in H^{1}(\Omega) \cap L^{\infty}(\Omega) .
$$

Then, if we choose

$$
\psi(\mathbf{x})= \begin{cases}1-v(\mathbf{x}) & \mathbf{x} \in \Omega_{1} \\ -v(\mathbf{x}) & \mathbf{x} \in \Omega_{2} \\ 0 & \text { elsewhere }\end{cases}
$$

we obtain

$$
\begin{aligned}
b_{\gamma_{B}}(u ; v, \psi)= & \int_{\Omega_{1}}\left[v(1-v)|\nabla u|^{2}-\alpha(v-1)^{2}-\varepsilon|\nabla v|^{2}\right] d \mathbf{x} \\
& -\int_{\Omega_{2}}\left[v^{2}|\nabla u|^{2}+\alpha(v-1) v+\varepsilon|\nabla v|^{2}\right] d \mathbf{x} \\
& -\frac{1}{\gamma_{B}} \int_{C R_{k-1} \cap \Omega_{2}} v^{2} d \mathbf{x}+\frac{1}{\gamma_{B}} \int_{C R_{k-1} \cap \Omega_{1}} v(1-v) d \mathbf{x}=0 .
\end{aligned}
$$

The left-hand side of (2.8) consists of four negative terms, leading to a contradiction.

2.1. The finite element discretization. We introduce the discrete counterpart of the minimization problem (2.5) in a finite element setting. Thus, we denote by $\left\{\mathcal{T}_{h}\right\}_{h>0}$ a family of meshes of the domain $\bar{\Omega}$, with $N_{h}$ the index set of the vertices of $\mathcal{T}_{h}$ and $\mathcal{E}_{h}$ the skeleton of $\mathcal{T}_{h}$. Henceforth, we assume that the boundary of $\Omega_{D^{ \pm}}$ 
coincides with the union of consecutive edges in $\mathcal{E}_{h}$. With $\left\{\mathcal{T}_{h}\right\}_{h>0}$ we associate the space $X_{h}$ of the continuous piecewise linear finite elements [15].

We denote by $I_{h}\left(u_{h}, v_{h}\right)$ the discrete correspondent of $I(u, v)$ in $(2.6)$, given by

$$
\begin{aligned}
I_{h}\left(u_{h}, v_{h}\right) & =\int_{\Omega}\left[\left(P_{h}\left(v_{h}^{2}\right)+\eta\right)\left|\nabla u_{h}\right|^{2} d \mathbf{x}+\alpha P_{h}\left(\left(1-v_{h}\right)^{2}\right)+\varepsilon\left|\nabla v_{h}\right|^{2}\right] d \mathbf{x} \\
& +\frac{1}{\gamma_{A}} \int_{\Omega_{D^{ \pm}}} P_{h}\left(\left(g_{h}\left(t_{k}\right)-u_{h}\right)^{2}\right) d \mathbf{x}+\frac{1}{\gamma_{B}} \int_{C R_{k-1}} P_{h}\left(v_{h}^{2}\right) d \mathbf{x},
\end{aligned}
$$

where $P_{h}: C^{0}(\bar{\Omega}) \rightarrow X_{h}$ is the Lagrangian interpolant onto the space $X_{h}$, with $g_{h}\left(t_{k}\right) \in X_{h}$ a suitable discrete approximation of $g\left(t_{k}\right)$. In particular, we pick $g_{h}\left(t_{k}\right)$ such that

$$
\int_{\Omega_{D^{ \pm}}} g_{h}\left(t_{k}\right) w_{h} d \mathbf{x}=\int_{\Omega_{D^{ \pm}}} g\left(t_{k}\right) w_{h} d \mathbf{x} \quad \forall w_{h} \in X_{h}
$$

i.e., $g_{h}\left(t_{k}\right)$ is the $L^{2}\left(\Omega_{D^{ \pm}}\right)$-projection of $g\left(t_{k}\right)$ onto $X_{h}$. The action of the operator $P_{h}$ is equivalent to a mass lumping [41] and it allows us to extend Proposition 2.3 to the critical points of $I_{h}(\cdot, \cdot)$ as well.

In the sequel, we assume that the off-diagonal entries of the stiffness matrix $K=\left[k_{i j}\right]$ associated with the space $X_{h}$ be non-positive, i.e.,

$$
k_{i j}=\int_{\Omega} \nabla \xi_{i} \cdot \nabla \xi_{j} d \mathbf{x} \leq 0 \quad \forall i \neq j \in N_{h},
$$

where $\left\{\xi_{l}\right\}_{l=1}^{\# N_{h}}$ denotes the finite element basis of $X_{h}$. This condition is related to discrete maximum principle as discussed, for instance, in [16, 31, 40]. The discrete analogue to $(2.5)$ is

$$
\left(u_{h}\left(t_{k}\right), v_{h}\left(t_{k}\right)\right) \in \underset{\substack{\widehat{u}_{h} \in X_{h}, \widehat{v}_{h} \in X_{h}}}{\arg \min } I_{h}\left(\widehat{u}_{h}, \widehat{v}_{h}\right) .
$$

Analogously to Definition 2.2, we have the following

Definition 2.4. The pair $\left(u_{h}, v_{h}\right) \in X_{h} \times X_{h}$ is a critical point of $I_{h}(\cdot, \cdot)$ if $I_{h}^{\prime}\left(u_{h}, v_{h} ; \varphi_{h}, \psi_{h}\right)=0$ for all $\left(\varphi_{h}, \psi_{h}\right) \in X_{h} \times X_{h}$, where

$$
\begin{aligned}
I_{h}^{\prime}\left(u_{h}, v_{h} ; \varphi_{h}, \psi_{h}\right)= & 2\left(\int_{\Omega}\left(P_{h}\left(v_{h}^{2}\right)+\eta\right) \nabla u_{h} \cdot \nabla \varphi_{h} d \mathbf{x}+\frac{1}{\gamma_{A}} \int_{\Omega_{D^{ \pm}}} P_{h}\left(\left(u_{h}-g_{h}\left(t_{k}\right)\right) \varphi_{h}\right) d \mathbf{x}\right) \\
& +2\left(\int_{\Omega}\left[P_{h}\left(v_{h} \psi_{h}\right)\left|\nabla u_{h}\right|^{2}+\alpha P_{h}\left(\left(v_{h}-1\right) \psi_{h}\right)+\varepsilon \nabla v_{h} \cdot \nabla \psi_{h}\right] d \mathbf{x}\right. \\
& \left.+\frac{1}{\gamma_{B}} \int_{C R_{k-1}^{h}} P_{h}\left(v_{h} \psi_{h}\right) d \mathbf{x}\right)=: 2 a_{\gamma_{A}}^{h}\left(v_{h} ; u_{h}, \varphi_{h}\right)+2 b_{\gamma_{B}}^{h}\left(u_{h} ; v_{h}, \psi_{h}\right) .
\end{aligned}
$$

Proposition 2.3 can be adapted to the discrete case, suitably relying on assumption (2.11) and the properties of $P_{h}$ as shown in the following

Proposition 2.5. Let $\left(u_{h}, v_{h}\right) \in X_{h} \times X_{h}$ be a critical point of $I_{h}(\cdot, \cdot)$, then $0 \leq v_{h} \leq 1$ for all $x \in \Omega$.

Proof. The proof generalizes Proposition 2.2 in [10], by properly including the term $\int_{C R_{k-1}} P_{h}\left(v_{h}^{2}\right) d \mathbf{x}$. By mimicking in a discrete setting the proof of Proposition 2.3 , we suppose, by contradiction, that there exist two index sets $J_{1}, J_{2} \subset N_{h}$ 
where $v_{i}>1$ for all $i \in J_{1}$ and $v_{j}<0$ for all $j \in J_{2}$, where we let $v_{i}=v_{h}\left(\mathbf{x}_{i}, t_{k}\right)$.

Consider $j \in J_{2}$ such that $v_{j} \leq v_{i}$, for all $i \in N_{h}$ and let $\Delta_{j}$ be the patch of elements associated with $\mathbf{x}_{j}$ with $M_{j}=\left\{i \in N_{h}: \mathbf{x}_{i} \in \Delta_{j}\right\}$.

Now, if we choose as a test function the hat function $\xi_{j}$ associated with $\mathbf{x}_{j}$, from the equality $b_{\gamma_{B}}^{h}\left(u_{h} ; v_{h}, \psi_{h}\right)=0$ we have

$$
\begin{aligned}
\varepsilon \int_{\Delta_{j}} \nabla v_{h} \cdot \nabla \xi_{j} d \mathbf{x}= & -\int_{\Delta_{j}}\left[P_{h}\left(v_{h} \xi_{j}\right)\left|\nabla u_{h}\right|^{2}-\alpha P_{h}\left(\left(v_{h}-1\right) \xi_{j}\right)\right] d \mathbf{x} \\
& -\frac{1}{\gamma_{B}} \int_{C R_{k-1} \cap \Delta_{j}} P_{h}\left(v_{h} \xi_{j}\right) d \mathbf{x} \\
> & -\left.v_{j} \sum_{K \in \Delta_{j}}\left|\nabla u_{h}\right|_{K}\right|^{2} \frac{|K|}{3}-\alpha\left(v_{j}-1\right) \frac{\left|\Delta_{j}\right|}{3}>0
\end{aligned}
$$

where the last inequality is obtained considering that $v_{j}<0$ and that $P_{h}\left(v_{h} \xi_{j}\right)$ is a non-positive function on the set $C R_{k-1} \cap \Delta_{j}$. Since $v_{h}=\sum_{i \in N_{h}} v_{i} \xi_{i}$, and the sum of the rows of the stiffness matrix is zero, we have

$\varepsilon \int_{\Delta_{j}} \nabla v_{h} \cdot \nabla \xi_{j} d \mathbf{x}=\varepsilon \sum_{i \in M_{j}} k_{j i} v_{i}=\varepsilon \sum_{i \in M_{j}} k_{j i}\left(v_{i}-v_{j}\right)+\varepsilon \sum_{i \in M_{j}} k_{j i} v_{j}=\varepsilon \sum_{i \in M_{j}} k_{j i}\left(v_{i}-v_{j}\right)$.

Thus, using assumption (2.11) and the hypothesis $v_{j} \leq v_{i}$, we have that

$$
\varepsilon \int_{\Delta_{j}} \nabla v_{h} \cdot \nabla \xi_{j} d \mathbf{x} \leq 0
$$

in contradiction with (2.12).

Similarly, we can proceed to contradict the existence of nodes in $J_{1}$. Consider $j \in J_{1}$ such that $v_{j} \geq v_{i}$ for all $i \in N_{h}$ and let $\Delta_{j}$ be the patch of elements associated with $\mathbf{x}_{j}$. Now, choosing again as a test function the hat function $\xi_{j}$ associated with $\mathbf{x}_{j}$, from the equality $b_{\gamma_{B}}^{h}\left(u_{h} ; v_{h}, \psi_{h}\right)=0$ we have

$$
\begin{aligned}
\varepsilon \int_{\Delta_{j}} \nabla v_{h} \cdot \nabla \xi_{j} d \mathbf{x}= & -\int_{\Delta_{j}}\left[P_{h}\left(v_{h} \xi_{j}\right)\left|\nabla u_{h}\right|^{2}-\alpha P_{h}\left(\left(v_{h}-1\right) \xi_{j}\right)\right] d \mathbf{x} \\
& -\frac{1}{\gamma_{B}} \int_{C R_{k-1} \cap \Delta_{j}} P_{h}\left(v_{h} \xi_{j}\right) d \mathbf{x} \\
< & -\left.v_{j} \sum_{K \in \Delta_{j}}\left|\nabla u_{h}\right|_{K}\right|^{2} \frac{|K|}{3}-\alpha\left(v_{j}-1\right) \frac{\left|\Delta_{j}\right|}{3}<0,
\end{aligned}
$$

where the last inequality is obtained considering that $v_{j}>1$ and that $P_{h}\left(v_{h} \xi_{j}\right)$ is a positive function on the set $C R_{k-1} \cap \Delta_{j}$. Following a similar reasoning as before and thanks to the hypothesis $v_{j} \geq v_{i}$, we have that $\varepsilon \int_{\Delta_{j}} \nabla v_{h} \cdot \nabla \xi_{j} d \mathbf{x} \geq 0$ in contradiction with (2.13).

3. An anisotropic error estimator for the modified Ambrosio-Tortorelli functional. Goal of this section is to provide a suitable optimization procedure for minimizing functional (2.4) by successive minimizations of (2.9) on adapted anisotropic meshes. For this purpose, we first lay down the anisotropic background and then we derive an a posteriori estimator for $\left|I^{\prime}\left(u_{h}, v_{h} ; \varphi, \psi\right)\right|$, by extending the analysis in [10]. 
3.1. The anisotropic background. We refer to the setting in [21, 34], where the anisotropic information is derived from the spectral properties of the standard affine $\operatorname{map} T_{K}: \widehat{K} \rightarrow K$ with

$$
\mathbf{x}=T_{K}(\widehat{\mathbf{x}})=M_{K} \widehat{\mathbf{x}}+\mathbf{t}_{K}
$$

between the equilateral reference triangle $\widehat{K}$ inscribed in the unit circle and the generic triangle $K$ of the mesh $\mathcal{T}_{h}$, with $M_{K} \in \mathbb{R}^{2 \times 2}, \mathbf{t}_{K} \in \mathbb{R}^{2}, \mathbf{x} \in K, \widehat{\mathbf{x}} \in \widehat{K}$.

We introduce the polar decomposition of the Jacobian $M_{K}$, i.e., $M_{K}=B_{K} Z_{K}$, where $B_{K}, Z_{K} \in \mathbb{R}^{2 \times 2}$ are a symmetric positive definite and an orthogonal matrix, respectively. The first matrix models the deformation of $K$, while $Z_{K}$ rotates it rigidly. Then, we consider the eigenvalue factorization of $B_{K}$ as $B_{K}=R_{K}^{T} \Lambda_{K} R_{K}$, with $R_{K}^{T}=\left[\mathbf{r}_{1, K}, \mathbf{r}_{2, K}\right]$ and $\Lambda_{K}=\operatorname{diag}\left(\lambda_{1, K}, \lambda_{2, K}\right)$. In particular, the eigenvectors $\mathbf{r}_{1, K}$, $\mathbf{r}_{2, K}$ give the directions of the semi-axes of the ellipse circumscribed to $K$, while the eigevalues $\lambda_{1, K}, \lambda_{2, K}$ measure the length of these semi-axes (see Figure 3.1). We also define the aspect ratio of the element $K$ by $s_{K}=\lambda_{1, K} / \lambda_{2, K}$.

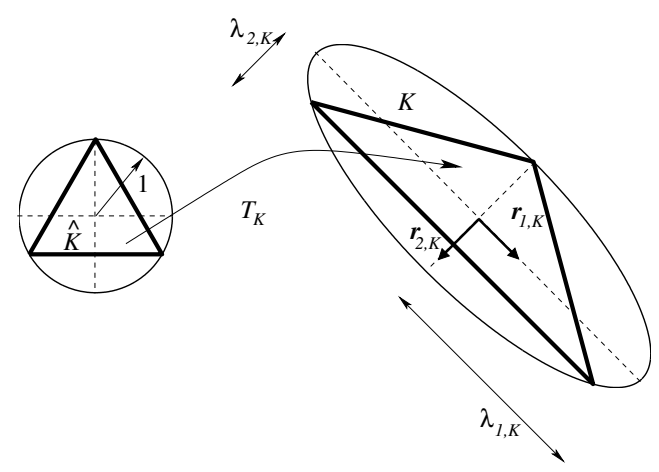

FIG. 3.1. Geometric quantities associated with the map $T_{K}$

With a view to an anisotropic control of the mesh, we introduce the quasiinterpolant Clément operator $Q_{h}: L^{2}(\Omega) \rightarrow X_{h}$ [17]. We recall the following anisotropic estimate for the interpolation error.

LEMma 3.1. Let $w \in H^{1}(\Omega)$. If the cardinality $\# \Delta_{K} \leq \mathcal{N}$ for some $\mathcal{N} \in \mathbb{N}$, and $\operatorname{diam}\left(T_{K}^{-1}\left(\Delta_{K}\right)\right) \leq C_{\Delta} \simeq O(1)$, where $\Delta_{K}=\left\{T \in \mathcal{T}_{h}: T \cap K \neq \emptyset\right\}$, then there exist constants $C_{s}=C_{s}\left(\mathcal{N}, C_{\Delta}\right)$, with $s=0,1,2$, such that, for any $K \in \mathcal{T}_{h}$, it holds

$$
\left\|w-Q_{h}(w)\right\|_{H^{s}(K)} \leq C_{s}\left(\frac{1}{\lambda_{2, K}}\right)^{s}\left[\sum_{i=1}^{2} \lambda_{i, K}^{2}\left(\mathbf{r}_{i, K}^{T} G_{\Delta_{K}}(w) \mathbf{r}_{i, K}\right)\right]^{1 / 2},
$$

with $s=0,1$, and

$$
\left\|w-Q_{h}(w)\right\|_{L^{2}(\partial K)} \leq C_{2}\left(\frac{h_{K}}{\lambda_{1, K} \lambda_{2, K}}\right)^{1 / 2}\left[\sum_{i=1}^{2} \lambda_{i, K}^{2}\left(\mathbf{r}_{i, K}^{T} G_{\Delta_{K}}(w) \mathbf{r}_{i, K}\right)\right]^{1 / 2}
$$

where $h_{K}=\operatorname{diam}(K)$,

$$
G_{\Delta_{K}}(w)=\sum_{T \in \Delta_{K}} G_{T}(w)
$$


is a symmetric positive semi-definite matrix with

$$
G_{T}(w)=\left[\begin{array}{cc}
\int_{T}\left(\frac{\partial w}{\partial x_{1}}\right)^{2} d \mathbf{x} & \int_{T} \frac{\partial w}{\partial x_{1}} \frac{\partial w}{\partial x_{2}} d \mathbf{x} \\
\int_{T} \frac{\partial w}{\partial x_{1}} \frac{\partial w}{\partial x_{2}} d \mathbf{x} & \int_{T}\left(\frac{\partial w}{\partial x_{2}}\right)^{2} d \mathbf{x}
\end{array}\right]
$$

for any $T \in \mathcal{T}_{h}$.

Proof. See [23, 24] for the details.

Notice that the geometrical hypotheses in Lemma 3.1 do not limit explicitly the anisotropic features (stretching factor and orientation) of each element, but rather they ensure some smoothness in the variation of the anisotropic features [36].

We recall an equivalence result between the standard $H^{1}\left(\Delta_{K}\right)$-seminorm and its anisotropic correspondent:

Lemma 3.2. Let $w \in H^{1}(\Omega)$ and $K \in \mathcal{T}_{h}$. For any $\beta_{1}, \beta_{2}>0$, it holds

$$
\min \left\{\beta_{1}, \beta_{2}\right\} \leq \frac{\beta_{1}\left(\mathbf{r}_{1, K}^{T} G_{\Delta_{K}}(w) \mathbf{r}_{1, K}\right)+\beta_{2}\left(\mathbf{r}_{2, K}^{T} G_{\Delta_{K}}(w) \mathbf{r}_{2, K}\right)}{|w|_{H^{1}\left(\Delta_{K}\right)}^{2}} \leq \max \left\{\beta_{1}, \beta_{2}\right\},
$$

where $G_{\Delta_{K}}(\cdot)$ is defined as in (3.3).

Proof. See [33] for the details.

3.2. An a posteriori error estimator. We can now state the main result of this section which represents the anisotropic analogue of Proposition 3.1 in [10].

Proposition 3.3. Let $\left(u_{h}, v_{h}\right) \in X_{h} \times X_{h}$ be the critical point of $I_{h}(\cdot, \cdot)$ according to Definition 2.4. Then, it holds

$\left|I^{\prime}\left(u_{h}, v_{h} ; \varphi, \psi\right)\right| \leq C \sum_{K \in \mathcal{T}_{h}}\left\{\rho_{K}^{A}\left(v_{h}, u_{h}\right) \omega_{K}(\varphi)+\rho_{K}^{B}\left(u_{h}, v_{h}\right) \omega_{K}(\psi)\right\} \quad \forall \varphi, \psi \in H^{1}(\Omega)$,

where $C=C\left(\mathcal{N}, C_{\Delta}\right)$, while

$$
\begin{aligned}
\rho_{K}^{A}\left(v_{h}, u_{h}\right)= & \frac{1}{2}\left\|\llbracket \nabla u_{h} \rrbracket\right\|_{L^{\infty}(\partial K)}\left\|v_{h}^{2}+\eta\right\|_{L^{2}(\partial K)}\left(\frac{h_{K}}{\lambda_{1, K} \lambda_{2, K}}\right)^{\frac{1}{2}} \\
& +\left\|2 v_{h}\left(\nabla v_{h} \cdot \nabla u_{h}\right)\right\|_{L^{2}(K)}+\frac{\delta_{K, \Omega_{D}^{ \pm}}}{\gamma_{A}}\left(\left\|u_{h}-g_{h}\left(t_{k}\right)\right\|_{L^{2}(K)}\right. \\
& \left.+\left\|g_{h}\left(t_{k}\right)-g\left(t_{k}\right)\right\|_{L^{2}(K)}\right)+\frac{1}{\lambda_{2, K}}\left[\left\|v_{h}^{2}-P_{h}\left(v_{h}^{2}\right)\right\|_{L^{\infty}(K)}\left\|\nabla u_{h}\right\|_{L^{2}(K)}\right. \\
& \left.+\frac{|K|^{1 / 2} h_{K}^{2}}{\gamma_{A}}\left|u_{h}-g_{h}\left(t_{k}\right)\right|_{W^{1, \infty}(K)}\right] \\
\rho_{K}^{B}\left(u_{h}, v_{h}\right)= & \left\|\left(\left|\nabla u_{h}\right|^{2}+\alpha\right) v_{h}-\alpha\right\|_{L^{2}(K)}+\frac{\varepsilon}{2}\left\|\llbracket \nabla v_{h} \rrbracket\right\|_{L^{2}(\partial K)}\left(\frac{h_{K}}{\lambda_{1, K} \lambda_{2, K}}\right)^{1 / 2} \\
& +\frac{\delta_{K, C R_{k-1}}\left\|v_{h}\right\|_{L^{2}(K)}+\frac{h_{K}^{2}}{\lambda_{2, K}}\left[\left\|\left|\nabla u_{h}\right|^{2}+\alpha\right\|_{L^{2}(K)}\right.}{}+\frac{\left.|K|^{1 / 2} \delta_{K, C R_{k-1}}\right]\left|v_{h}\right|_{W^{1, \infty}(K)}}{\gamma_{B}} \quad \forall w \in H^{1}(\Omega), \\
\omega_{K}(w)= & {\left[\sum_{i=1}^{2} \lambda_{i, K}^{2}\left(\mathbf{r}_{i, K}^{T} G_{\Delta_{K}}(w) \mathbf{r}_{i, K}\right)\right]^{1 / 2} \quad \forall w }
\end{aligned}
$$


where

$$
\llbracket w_{h} \rrbracket= \begin{cases}\left|\left[\nabla w_{h} \cdot \nu\right]\right| & \text { on } \mathcal{E}_{h} \\ \left|\nabla w_{h} \cdot \nu\right| & \text { on } \mathcal{E}_{h} \cap \partial \Omega\end{cases}
$$

denotes the absolute value of the jump of the normal derivative, with $\nu$ the unit normal vector to the generic edge in $\mathcal{E}_{h}, g_{h}$ is chosen as in (2.10) and $\delta_{K, \varpi}$ is such that $\delta_{K, \varpi}=1$ if $K \cap \varpi \neq \emptyset$ and $\delta_{K, \varpi}=0$ otherwise.

Proof. Since $\left(u_{h}, v_{h}\right)$ is a critical point of $I_{h}(\cdot, \cdot)$, we have

$$
a_{\gamma_{A}}^{h}\left(v_{h} ; u_{h}, \varphi_{h}\right)=0 \quad \forall \varphi_{h} \in X_{h}, \quad b_{\gamma_{B}}^{h}\left(u_{h} ; v_{h}, \psi_{h}\right)=0 \quad \forall \psi_{h} \in X_{h} .
$$

Moreover, from (2.7), for any pair $(\varphi, \psi) \in H^{1}(\Omega) \times H^{1}(\Omega)$, it holds

$$
\left|I^{\prime}\left(u_{h}, v_{h} ; \varphi, \psi\right)\right| \leq 2\left|a_{\gamma_{A}}\left(v_{h} ; u_{h}, \varphi\right)\right|+2\left|b_{\gamma_{B}}\left(u_{h} ; v_{h}, \psi\right)\right| .
$$

Let us deal with the two terms above, separately. We start from $\left|a_{\gamma_{A}}\left(v_{h} ; u_{h}, \varphi\right)\right|$. Thanks to (3.8), we have

$$
\begin{aligned}
\left|a_{\gamma_{A}}\left(v_{h} ; u_{h}, \varphi\right)\right| & \leq\left|a_{\gamma_{A}}\left(v_{h} ; u_{h}, \varphi-\varphi_{h}\right)\right| \\
& +\left|a_{\gamma_{A}}\left(v_{h} ; u_{h}, \varphi_{h}\right)-a_{\gamma_{A}}^{h}\left(v_{h} ; u_{h}, \varphi_{h}\right)\right| \quad \forall \varphi \in H^{1}(\Omega), \quad \forall \varphi_{h} \in X_{h} .
\end{aligned}
$$

Concerning the first term on the right-hand side of (3.10), we get

$$
\begin{aligned}
\left|a_{\gamma_{A}}\left(v_{h} ; u_{h}, \varphi-\varphi_{h}\right)\right| & \left|\sum_{K \in \mathcal{T}_{h}}\left\{\int_{K}\left(v_{h}^{2}+\eta\right) \nabla u_{h} \cdot \nabla\left(\varphi-\varphi_{h}\right) d \mathbf{x}+\frac{1}{\gamma_{A}} \int_{K}\left(u_{h}-g\left(t_{k}\right)\right)\left(\varphi-\varphi_{h}\right) \chi_{\Omega_{D}^{ \pm}} d \mathbf{x}\right\}\right| \\
= & \mid \sum_{K \in \mathcal{T}_{h}}\left\{\int_{K}-2 v_{h}\left(\nabla v_{h} \cdot \nabla u_{h}\right)\left(\varphi-\varphi_{h}\right) d \mathbf{x}+\int_{\partial K}\left(v_{h}^{2}+\eta\right) \nabla u_{h} \cdot \nu\left(\varphi-\varphi_{h}\right) d s\right. \\
& \left.+\frac{1}{\gamma_{A}} \int_{K}\left[\left(u_{h}-g_{h}\left(t_{k}\right)\right)+\left(g_{h}\left(t_{k}\right)-g\left(t_{k}\right)\right)\right]\left(\varphi-\varphi_{h}\right) \chi_{\Omega_{D}^{ \pm}} d \mathbf{x}\right\} \mid \\
\leq & \sum_{K \in \mathcal{T}_{h}}\left\{\left\|2 v_{h}\left(\nabla v_{h} \cdot \nabla u_{h}\right)\right\|_{L^{2}(K)}\left\|\varphi-\varphi_{h}\right\|_{L^{2}(K)}+\frac{1}{2} \int_{\partial K} \llbracket \nabla u_{h} \rrbracket\left|v_{h}^{2}+\eta\right|\left|\varphi-\varphi_{h}\right| d s\right. \\
+ & \left.\frac{1}{\gamma_{A}}\left(\left\|\left(u_{h}-g_{h}\left(t_{k}\right)\right) \chi_{\Omega_{D}^{ \pm}}\right\|_{L^{2}(K)}+\left\|\left(g_{h}\left(t_{k}\right)-g\left(t_{k}\right)\right) \chi_{\Omega_{D}^{ \pm}}\right\|_{L^{2}(K)}\right)\left\|\left(\varphi-\varphi_{h}\right) \chi_{\Omega_{D}^{ \pm}}\right\|_{L^{2}(K)}\right\} \\
\leq & \sum_{K \in \mathcal{T}_{h}}\left\{\left\|2 v_{h}\left(\nabla v_{h} \cdot \nabla u_{h}\right)\right\|_{L^{2}(K)}\left\|\varphi-\varphi_{h}\right\|_{L^{2}(K)}\right. \\
& +\frac{1}{2}\left\|\llbracket \nabla u_{h} \rrbracket\right\|_{L^{\infty}(\partial K)}\left\|v_{h}^{2}+\eta\right\|_{L^{2}(\partial K)}\left\|\varphi-\varphi_{h}\right\|_{L^{2}(\partial K)}+\frac{1}{\gamma_{A}}\left(\left\|\left(u_{h}-g_{h}\left(t_{k}\right)\right) \chi_{\Omega_{D}^{ \pm}}\right\|_{L^{2}(K)}\right) \\
& \left.\left.+\left\|\left(g_{h}\left(t_{k}\right)-g\left(t_{k}\right)\right) \chi_{\Omega_{D}^{ \pm}}\right\|_{L^{2}(K)}\right)\left\|\left(\varphi-\varphi_{h}\right) \chi_{\Omega_{D}^{ \pm}}\right\|_{L^{2}(K)}\right\},
\end{aligned}
$$

after splitting the integrals on the mesh elements, exploiting integration by parts, Hölder and Cauchy-Schwarz inequalities, and definition (3.7). Hereafter, $\chi_{\varpi}$ denotes the characteristic function of the set $\varpi$. Picking $\varphi_{h}=Q_{h}(\varphi)$ and thanks to Lemma 3.1 
with $s=0$, we obtain

$$
\begin{aligned}
& \left|a_{\gamma_{A}}\left(v_{h} ; u_{h}, \varphi-\varphi_{h}\right)\right| \\
& \leq C \sum_{K \in \mathcal{T}_{h}}\left\{\left\|2 v_{h}\left(\nabla v_{h} \cdot \nabla u_{h}\right)\right\|_{L^{2}(K)}+\frac{1}{2}\left\|\llbracket \nabla u_{h} \rrbracket\right\|_{L^{\infty}(\partial K)}\left\|v_{h}^{2}+\eta\right\|_{L^{2}(\partial K)}\left(\frac{h_{K}}{\lambda_{1, K} \lambda_{2, K}}\right)^{\frac{1}{2}}\right. \\
& \left.+\frac{\delta_{K, \Omega_{D}^{ \pm}}}{\gamma_{A}}\left(\left\|u_{h}-g_{h}\left(t_{k}\right)\right\|_{L^{2}(K)}+\left\|g_{h}\left(t_{k}\right)-g\left(t_{k}\right)\right\|_{L^{2}(K)}\right)\right\}\left[\sum_{i=1}^{2} \lambda_{i, K}^{2}\left(\mathbf{r}_{i, K}^{T} G_{\Delta_{K}}(\varphi) \mathbf{r}_{i, K}\right)\right]^{\frac{1}{2}} .
\end{aligned}
$$

Let us now deal with the second term on the right-hand side of (3.10). We anticipate the auxiliary result

$$
\left|w_{h} \varphi_{h}\right|_{H^{2}(K)} \leq 4\left|w_{h}\right|_{W^{1, \infty}(K)}\left\|\nabla \varphi_{h}\right\|_{L^{2}(K)} \quad \forall w_{h}, \varphi_{h} \in X_{h}, \quad \forall K \in \mathcal{T}_{h},
$$

which can be proved by straightforward calculus. Now, employing standard inequalities (Hölder, Cauchy-Schwarz) together with the definition of $g_{h}\left(t_{k}\right)$ and the standard isotropic estimate for the $L^{2}$-norm of the interpolation error associated with $P_{h}$, we get

$$
\begin{aligned}
\mid a_{\gamma_{A}}\left(v_{h} ; u_{h}, \varphi_{h}\right)- & a_{\gamma_{A}}^{h}\left(v_{h} ; u_{h}, \varphi_{h}\right)|\leq| \int_{\Omega}\left[v_{h}^{2}-P_{h}\left(v_{h}^{2}\right)\right] \nabla u_{h} \cdot \nabla \varphi_{h} d \mathbf{x} \mid \\
& +\frac{1}{\gamma_{A}}\left|\int_{\Omega_{D}^{ \pm}}\left[\left(u_{h}-g_{h}\left(t_{k}\right)\right) \varphi_{h}-P_{h}\left(\left(u_{h}-g_{h}\left(t_{k}\right)\right) \varphi_{h}\right)\right] d \mathbf{x}\right| \\
& +\frac{1}{\gamma_{A}}\left|\int_{\Omega_{D}^{ \pm}}\left(g_{h}\left(t_{k}\right) \varphi_{h}-g\left(t_{k}\right) \varphi_{h}\right) d \mathbf{x}\right| \\
\leq & C \sum_{K \in \mathcal{T}_{h}}\left\{\left\|v_{h}^{2}-P_{h}\left(v_{h}^{2}\right)\right\|_{L^{\infty}(K)}\left\|\nabla u_{h}\right\|_{L^{2}(K)}\left\|\nabla \varphi_{h}\right\|_{L^{2}(K)}\right. \\
& \left.+\frac{|K|^{1 / 2} h_{K}^{2}}{\gamma_{A}}\left|\left(u_{h}-g_{h}\left(t_{k}\right)\right) \varphi_{h}\right|_{H^{2}(K)}\right\},
\end{aligned}
$$

where the constant $C$ does not depend on the aspect ratio $s_{K}$ of $K$. Then, we employ (3.13) together with estimate (3.1) with $s=1$ and Lemma 3.2 with $\beta_{1}=\lambda_{1, K}^{2}$, $\beta_{2}=\lambda_{2, K}^{2}$, to obtain

$$
\begin{aligned}
&\left|a_{\gamma_{A}}\left(v_{h} ; u_{h}, \varphi_{h}\right)-a_{\gamma_{A}}^{h}\left(v_{h} ; u_{h}, \varphi_{h}\right)\right| \begin{aligned}
\leq C \sum_{K \in \mathcal{T}_{h}}\{ & \left\|v_{h}^{2}-P_{h}\left(v_{h}^{2}\right)\right\|_{L^{\infty}(K)}\left\|\nabla u_{h}\right\|_{L^{2}(K)}\left\|\nabla \varphi_{h}\right\|_{L^{2}(K)} \\
& \left.+\frac{|K|^{1 / 2} h_{K}^{2}}{\gamma_{A}}\left|u_{h}-g_{h}\left(t_{k}\right)\right|_{W^{1, \infty}(K)}\left\|\nabla \varphi_{h}\right\|_{L^{2}(K)}\right\}
\end{aligned} \\
& \leq C \sum_{K \in \mathcal{T}_{h}}\left\{\left(\left\|v_{h}^{2}-P_{h}\left(v_{h}^{2}\right)\right\|_{L^{\infty}(K)}\left\|\nabla u_{h}\right\|_{L^{2}(K)}+\frac{|K|^{1 / 2} h_{K}^{2}}{\gamma_{A}}\left|u_{h}-g_{h}\left(t_{k}\right)\right|_{W^{1, \infty}(K)}\right)\right. \\
&\left.\quad\left(\left\|\nabla \varphi_{h}-\nabla \varphi\right\|_{L^{2}(K)}+\|\nabla \varphi\|_{L^{2}(K)}\right)\right\} \\
& \leq C \sum_{K \in \mathcal{T}_{h}}\left\{\left(\left\|v_{h}^{2}-P_{h}\left(v_{h}^{2}\right)\right\|_{L^{\infty}(K)}\left\|\nabla u_{h}\right\|_{L^{2}(K)}+\frac{|K|^{1 / 2} h_{K}^{2}}{\gamma_{A}}\left|u_{h}-g_{h}\left(t_{k}\right)\right|_{W^{1, \infty}(K)}\right)\right. \\
&\left.\frac{1}{\lambda_{2, K}}\left[\sum_{i=1}^{2} \lambda_{i, K}^{2}\left(\mathbf{r}_{i, K}^{T} G_{\Delta_{K}}(\varphi) \mathbf{r}_{i, K}\right)\right]^{1 / 2}\right\} .
\end{aligned}
$$


Therefore, collecting (3.12) and (3.15), we are able to bound the first term on the right-hand side of (3.9), as

$$
\left|a_{\gamma_{A}}\left(v_{h} ; u_{h}, \varphi\right)\right| \leq C \sum_{K \in \mathcal{T}_{h}} \rho_{K}^{A}\left(v_{h}, u_{h}\right) \omega_{K}^{A}(\varphi)
$$

Let us consider now the second term on the righ-hand side of (3.9). In the same way as in (3.10) and thanks to (3.8), we have

$$
\begin{aligned}
\left|b_{\gamma_{B}}\left(u_{h} ; v_{h}, \psi\right)\right| \leq & \left|b_{\gamma_{B}}\left(u_{h} ; v_{h}, \psi-\psi_{h}\right)\right| \\
& +\left|b_{\gamma_{B}}\left(u_{h} ; v_{h}, \psi_{h}\right)-b_{\gamma_{B}}^{h}\left(u_{h} ; v_{h}, \psi_{h}\right)\right| \quad \forall \psi \in H^{1}(\Omega), \forall \psi_{h} \in X_{h} .
\end{aligned}
$$

We tackle the first term $\left|b_{\gamma_{B}}\left(u_{h} ; v_{h}, \psi-\psi_{h}\right)\right|$. Rewriting the integrals on $\Omega$ over the mesh elements, integrating by parts, and thanks to the Cauchy-Schwarz inequality and definition (3.7), we obtain

$$
\begin{aligned}
\left|b_{\gamma_{B}}\left(u_{h} ; v_{h}, \psi-\psi_{h}\right)\right| \\
=\mid \sum_{K \in \mathcal{T}_{h}}\left\{\int_{K}\left[\left(\left(\left|\nabla u_{h}\right|^{2}+\alpha\right) v_{h}-\alpha\right)\left(\psi-\psi_{h}\right)+\varepsilon \nabla v_{h} \cdot \nabla\left(\psi-\psi_{h}\right)\right] d \mathbf{x}\right. \\
\left.\quad+\frac{1}{\gamma_{B}} \int_{K} v_{h}\left(\psi-\psi_{h}\right) \chi_{C R_{k-1}} d \mathbf{x}\right\} \mid \\
\leq \sum_{K \in \mathcal{T}_{h}}\left\{\left\|\left(\left|\nabla u_{h}\right|^{2}+\alpha\right) v_{h}-\alpha\right\|_{L^{2}(K)}\left\|\psi-\psi_{h}\right\|_{L^{2}(K)}+\left|\varepsilon \int_{\partial K}\left(\psi-\psi_{h}\right) \nabla v_{h} \cdot \nu d s\right|\right. \\
\left.\quad+\frac{1}{\gamma_{B}}\left\|v_{h} \chi_{C R_{k-1}}\right\|_{L^{2}(K)}\left\|\left(\psi-\psi_{h}\right) \chi_{C R_{k-1}}\right\|_{L^{2}(K)}\right\} \\
\leq \sum_{K \in \mathcal{T}_{h}}\left\{\left\|\left(\left|\nabla u_{h}\right|^{2}+\alpha\right) v_{h}-\alpha\right\|_{L^{2}(K)}\left\|\psi-\psi_{h}\right\|_{L^{2}(K)}+\frac{\varepsilon}{2}\left\|\llbracket \nabla v_{h} \rrbracket\right\|_{L^{2}(\partial K)}\left\|\psi-\psi_{h}\right\|_{L^{2}(\partial K)}\right. \\
\left.\quad+\frac{1}{\gamma_{B}}\left\|v_{h} \chi_{C R_{k-1}}\right\|_{L^{2}(K)}\left\|\left(\psi-\psi_{h}\right) \chi_{C R_{k-1}}\right\|_{L^{2}(K)}\right\} .
\end{aligned}
$$

We now choose $\psi_{h}=Q_{h}(\psi)$ and use Lemma 3.1 to get

$$
\begin{aligned}
& \left|b_{\gamma_{B}}\left(u_{h} ; v_{h}, \psi-\psi_{h}\right)\right| \\
& \leq C \sum_{K \in \mathcal{T}_{h}}\left\{\left\|\left(\left|\nabla u_{h}\right|^{2}+\alpha\right) v_{h}-\alpha\right\|_{L^{2}(K)}+\frac{\varepsilon}{2}\left\|\llbracket \nabla v_{h} \rrbracket\right\|_{L^{2}(\partial K)}\left(\frac{h_{K}}{\lambda_{1, K} \lambda_{2, K}}\right)^{1 / 2}\right. \\
& \left.\quad+\frac{\delta_{K, C R_{k-1}}}{\gamma_{B}}\left\|v_{h}\right\|_{L^{2}(K)}\right\}\left[\sum_{i=1}^{2} \lambda_{i, K}^{2}\left(\mathbf{r}_{i, K}^{T} G_{\Delta_{K}}(\psi) \mathbf{r}_{i, K}\right)\right]^{1 / 2}
\end{aligned}
$$

We estimate now the second term on the right-hand side of (3.16). By mimicking the arguments employed in (3.14)-(3.15), we obtain the following bound:

$$
\begin{aligned}
& \left|b_{\gamma_{B}}\left(u_{h} ; v_{h}, \psi_{h}\right)-b_{\gamma_{B}}^{h}\left(u_{h} ; v_{h}, \psi_{h}\right)\right| \\
& \leq\left|\int_{\Omega}\left(v_{h} \psi_{h}-P_{h}\left(v_{h} \psi_{h}\right)\right)\left(\left|\nabla u_{h}\right|^{2}+\alpha\right) d \mathbf{x}\right|+\frac{1}{\gamma_{B}} \int_{C R_{k-1}}\left(v_{h} \psi_{h}-P_{h}\left(v_{h} \psi_{h}\right)\right) d \mathbf{x} \mid
\end{aligned}
$$




$$
\begin{gathered}
\leq C \sum_{K \in \mathcal{T}_{h}}\left\{\left\|v_{h} \psi_{h}-P_{h}\left(v_{h} \psi_{h}\right)\right\|_{L^{2}(K)}\left[\left\|\left|\nabla u_{h}\right|^{2}+\alpha\right\|_{L^{2}(K)}+\frac{|K|^{1 / 2} \delta_{K, C R_{k-1}}}{\gamma_{B}}\right]\right\} \\
\leq C \sum_{K \in \mathcal{T}_{h}}\left\{\left[\left\|\left|\nabla u_{h}\right|^{2}+\alpha\right\|_{L^{2}(K)}+\frac{|K|^{1 / 2} \delta_{K, C R_{k-1}}}{\gamma_{B}}\right] h_{K}^{2}\left|v_{h} \psi_{h}\right|_{H^{2}(K)}\right\} \\
\leq C \sum_{K \in \mathcal{T}_{h}}\left\{\left[\left\|\left|\nabla u_{h}\right|^{2}+\alpha\right\|_{L^{2}(K)}+\frac{|K|^{1 / 2} \delta_{K, C R_{k-1}}}{\gamma_{B}}\right] h_{K}^{2}\left|v_{h}\right|_{W^{1, \infty}(K)}\left\|\nabla \psi_{h}\right\|_{L^{2}(K)}\right\} \\
\leq C \sum_{K \in \mathcal{T}_{h}}\left\{\left[\left\|\left|\nabla u_{h}\right|^{2}+\alpha\right\|_{L^{2}(K)}+\frac{|K|^{1 / 2} \delta_{K, C R_{k-1}}}{\gamma_{B}}\right] h_{K}^{2}\left|v_{h}\right|_{W^{1, \infty}(K)}\right. \\
\left.\frac{1}{\lambda_{2, K}}\left[\sum_{i=1}^{2} \lambda_{i, K}^{2}\left(\mathbf{r}_{i, K}^{T} G_{\Delta_{K}}(\psi) \mathbf{r}_{i, K}\right)\right]^{1 / 2}\right\} .
\end{gathered}
$$

Inequalities (3.18) and (3.19) allow us to control the second term on the right-hand side of (3.9), i.e.,

$$
\left|b_{\gamma_{B}}\left(u_{h} ; v_{h}, \psi\right)\right| \leq C \sum_{K \in \mathcal{T}_{h}} \rho_{K}^{B}\left(u_{h}, v_{h}\right) \omega_{K}^{B}(\psi)
$$

Estimate (3.6) now follows in a straightforward way. $\square$

Estimate (3.6) holds for any choice of test functions $(\varphi, \psi) \in H^{1}(\Omega) \times H^{1}(\Omega)$. To get rid of the particular test functions, following [10], we can bound the dual norm $\left\|I^{\prime}\left(u_{h}, v_{h}\right)\right\|_{\left(H^{1}(\Omega) \times H^{1}(\Omega)\right)^{*}}$ as

$$
\begin{aligned}
& \left\|I^{\prime}\left(u_{h}, v_{h}\right)\right\|_{\left(H^{1}(\Omega) \times H^{1}(\Omega)\right)^{*}} \\
& \quad \leq C\left[\left(\sum_{K \in \mathcal{T}_{h}} \lambda_{1, K}^{2}\left(\rho_{K}^{A}\left(v_{h}, u_{h}\right)\right)^{2}\right)^{1 / 2}+\left(\sum_{K \in \mathcal{T}_{h}} \lambda_{1, K}^{2}\left(\rho_{K}^{B}\left(u_{h}, v_{h}\right)\right)^{2}\right)^{1 / 2}\right],
\end{aligned}
$$

where $\left(H^{1}(\Omega) \times H^{1}(\Omega)\right)^{*}$ is the dual space of $H^{1}(\Omega) \times H^{1}(\Omega)$ and $C=C\left(\mathcal{N}, C_{\Delta}\right)$. Indeed, thanks to Lemma 3.2 and the discrete Cauchy-Schwarz inequality, we have

$$
\begin{aligned}
\left\|I^{\prime}\left(u_{h}, v_{h}\right)\right\|_{\left(H^{1}(\Omega) \times H^{1}(\Omega)\right)^{*}}=\sup _{(\varphi, \psi) \in H^{1}(\Omega) \times H^{1}(\Omega)} & \frac{\left|I^{\prime}\left(u_{h}, v_{h} ; \varphi, \psi\right)\right|}{\left[\|\varphi\|_{H^{1}(\Omega)}^{2}+\|\psi\|_{H^{1}(\Omega)}^{2}\right]^{1 / 2}} \\
\leq C\left[\left(\sum_{K \in \mathcal{T}_{h}} \lambda_{1, K}^{2}\left(\rho_{K}^{A}\left(v_{h}, u_{h}\right)\right)^{2}\right)^{1 / 2}+\right. & \left.\left(\sum_{K \in \mathcal{T}_{h}} \lambda_{1, K}^{2}\left(\rho_{K}^{B}\left(u_{h}, v_{h}\right)\right)^{2}\right)^{1 / 2}\right] \\
& {\left[\frac{\left(\|\varphi\|_{H^{1}(\Omega)}+\|\psi\|_{H^{1}(\Omega)}\right)}{\left(\|\varphi\|_{H^{1}(\Omega)}^{2}+\|\psi\|_{H^{1}(\Omega)}^{2}\right)^{1 / 2}}\right] . }
\end{aligned}
$$

Nevertheless, the right-hand side of (3.20), despite being an explicitly computable quantity, turns out to be a very poor error estimator in terms of driving efficient anisotropic mesh adaptation. Thus, we cannot pursue the approach in [10]. Alternatively, we make a specific choice for the test function pair in (3.6), namely $\varphi=u$ and $\psi=v$. This is mainly motivated by the fact that we weight the residual $\rho_{K}^{A}\left(v_{h}, u_{h}\right)$ associated with the derivative of $I(\cdot, \cdot)$ with respect to $u$ with the directional information provided by $u$ itself, and analogously for $\rho_{K}^{B}\left(u_{h}, v_{h}\right)$ weighted via $v$. As a consequence, the error estimator that we propose is

$$
\eta\left(u_{h}, v_{h}\right)=\sum_{K \in \mathcal{T}_{h}} \eta_{K}\left(u_{h}, v_{h}\right),
$$


with $\eta_{K}\left(u_{h}, v_{h}\right)=\rho_{K}^{A}\left(v_{h}, u_{h}\right) \omega_{K}^{R}\left(u_{h}\right)+\rho_{K}^{B}\left(u_{h}, v_{h}\right) \omega_{K}^{R}\left(v_{h}\right)$, where

$$
\omega_{K}^{R}\left(z_{h}\right)=\left[\sum_{i=1}^{2} \lambda_{i, K}^{2}\left(\mathbf{r}_{i, K}^{T} G_{\Delta_{K}}^{R}\left(z_{h}\right) \mathbf{r}_{i, K}\right)\right]^{1 / 2} \quad \text { with } \quad z_{h}=u_{h}, v_{h},
$$

with $G_{\Delta_{K}}^{R}\left(z_{h}\right)$ the matrix $G_{\Delta_{K}}$ defined as in Lemma 3.1 applied to the recovered gradient from $z_{h}[42,34,35]$. Notice that we have already replaced $(\varphi, \psi)$ by the pair $\left(u_{h}, v_{h}\right)$ in (3.6) to make the estimator explicitly computable.

REMARK 3.1. In the numerical computations of the next section, after [10], we replace definition (2.2) by the discrete version

$$
C R_{k-1}^{h}=\bigcup_{e \in \mathcal{E}_{h}^{C R}} \bar{e}, \quad \text { where } \mathcal{E}_{h}^{C R}=\left\{e \in \mathcal{E}_{h}: v_{h}\left(\mathbf{x}, t_{k-1}\right) \leq \mathrm{CRTOL}, \forall \mathbf{x} \in \bar{e}\right\},
$$

which enjoys one-dimensional features. It is beyond the purpose of this paper to study the error induced by this approximation.

4. The numerical procedure. The numerical minimization of the functional (2.6) by successive minimizations of (2.9) is not a trivial task. In fact, the presence of the term $v^{2}|\nabla u|^{2}$ makes it nonconvex. Therefore, it is not possible, in general, to construct an algorithm with polynomial complexity guaranteeing convergence to the global minimizers. The methods in the literature in general only ensure convergence to local minima (see [4] and references therein).

In the first part of this section, we provide the algorithm employed for the minimization of $(2.6)$ in $H^{1}(\Omega) \times H^{1}(\Omega)$. The discrete version of this algorithm coupled with the mesh adaptive procedure is introduced in Section 4.2.

4.1. The minimization algorithm. To minimize (2.6), we resort to the alternate minimization algorithm proposed in [9]. This method exploits the convexity of the functional with respect to the two separate variables. Thus, after fixing a termination tolerance $\mathrm{VTOL} \ll 1$, the algorithm is the following:

Algorithm 1.

1. Set $k=0$;

2. If $k=0$, set $v^{1}=1$; else $v^{1}=v\left(t_{k-1}\right)$.

3. Set $i=1$; err $=1$;

while err $\geq$ VTOL $\boldsymbol{d o}$

4. $u^{i}=\arg \min I\left(z, v^{i}\right)$; $z \in H^{1}(\Omega)$

5. $v^{i+1}=\underset{z \in H^{1}(\Omega)}{\arg \min } I\left(u^{i}, z\right)$;

6. $\operatorname{err}=\left\|v^{i+1}-v^{i}\right\|_{L^{\infty}(\Omega)}$;

7. $i \leftarrow i+1$;

\section{end while}

8. $u\left(t_{k}\right)=u^{i-1} ; v\left(t_{k}\right)=v^{i}$;

9. $k \leftarrow k+1$;

10. if $k>F$, stop; else goto 2 .

Steps 4 . and 5 . involve the two separate convex minimizations.

In the literature, several examples of implementations of this algorithm are available (see, e.g., $[6,7,9,10]$ ) and a corresponding convergence proof can be obtained by exploiting [6, Theorem 1] and [10, Theorems 4.1 and 4.2]. 
With a view to the numerical implementation, we will consider the discrete counterpart of Algorithm 1. Since, in general, we expect the crack propagation to be a strongly anisotropic process, characterized by very steep gradients of both the fields $u$ and $v$, we will resort to a finite element discretization based on anisotropic adapted meshes, driven by the a posteriori error estimator derived in Proposition 3.3. The challenge is to properly merge the minimization algorithm with an anisotropic adaptive procedure, as shown in the next section.

4.2. The mesh adaptive procedure. Following [33, 34, 35], we use a metricbased mesh adaptive approach (see, e.g., [27]). In particular, for a fixed accuracy tolerance TOL, we "predict" the optimal mesh with the least number of elements.

A metric is a symmetric positive-definite tensor field $\mathcal{M}: \Omega \rightarrow \mathbb{R}^{2 \times 2}$ which, for any $\mathbf{x} \in \Omega$, provides the sizes that the optimal mesh should have along all the directions around $\mathbf{x}$. In practice, we approximate $\mathcal{M}$ via a piecewise constant metric on a given mesh $\mathcal{T}_{h}$, i.e., $\left.\mathcal{M}\right|_{K}=\mathcal{M}_{K}=\mathcal{R}_{K}^{T} \mathcal{L}_{K}^{-2} \mathcal{R}_{K}$, for any $K \in \mathcal{T}_{h}$, where the matrices $\mathcal{R}_{K}$ and $\mathcal{L}_{K}$ share the same structure as $R_{K}$ and $\Lambda_{K}$ in Section 3, respectively.

Actually, there exists a strict link between metrics and meshes. We can associate with an assigned mesh $\mathcal{T}_{h}$, a corresponding piecewise-constant metric identified by $M_{K}=R_{K}^{T} \Lambda_{K}^{-2} R_{K}$, for any $K \in \mathcal{T}_{h}$, where matrices $R_{K}$ and $\Lambda_{K}$ are exactly the same as in Section 3.1. Vice versa, for a given metric $\mathcal{M}$, we can build a mesh, say $\mathcal{T}_{\mathcal{M}}$, such that $\mathcal{M}_{K} \equiv M_{K}$, for any $K \in \mathcal{T}_{\mathcal{M}}$ (for all the details, we refer, for instance, to $[33,34])$.

The procedure we follow is first to derive a metric moving from the a posteriori error estimator (3.21) and then to generate the new mesh induced by this metric via a metric-based mesh generator. In particular, we resort to the function adaptmesh in FreeFem++ [30].

In the spirit of a standard predictive approach, the metric $\mathcal{M}$ is obtained via an iterative procedure. At each iteration, say $j$, we deal with three quantities:

i) the actual mesh $\mathcal{T}_{h}^{(j)}$;

ii) the new metric $\mathcal{M}^{(j+1)}$ computed on $\mathcal{T}_{h}^{(j)}$;

iii) the updated mesh $\mathcal{T}_{h}^{(j+1)}$ induced by $\mathcal{M}^{(j+1)}$.

The most tricky step is the prediction of the new metric out of the estimator $\eta\left(u_{h}, v_{h}\right)$. For this purpose, we suitably rewrite the local estimator $\eta_{K}\left(u_{h}, v_{h}\right)$ as

$$
\eta_{K}\left(u_{h}, v_{h}\right)=\mu_{K}\left\{\bar{\rho}_{K}^{A}\left(v_{h}, u_{h}\right) \bar{\omega}_{K}^{R}\left(u_{h}\right)+\bar{\rho}_{K}^{B}\left(u_{h}, v_{h}\right) \bar{\omega}_{K}^{R}\left(v_{h}\right)\right\},
$$

where $\mu_{K}=|\widehat{K}|\left(\lambda_{1, K} \lambda_{2, K}\right)^{3 / 2}$ gathers all the area $|K|$ information,

$$
\bar{\rho}_{K}^{A}\left(v_{h}, u_{h}\right)=\frac{\rho_{K}^{A}\left(v_{h}, u_{h}\right)}{\left(|\widehat{K}| \lambda_{1, K} \lambda_{2, K}\right)^{1 / 2}}, \quad \bar{\rho}_{K}^{B}\left(v_{h}, u_{h}\right)=\frac{\rho_{K}^{B}\left(v_{h}, u_{h}\right)}{\left(|\widehat{K}| \lambda_{1, K} \lambda_{2, K}\right)^{1 / 2}},
$$

are approximately pointwise values (at least for a sufficiently fine mesh), while the new weights

$$
\bar{\omega}_{K}^{R}\left(z_{h}\right)=\left[s_{K} \mathbf{r}_{1, K}^{T} \bar{G}_{\Delta_{K}}^{R}\left(z_{h}\right) \mathbf{r}_{1, K}+\frac{1}{s_{K}} \mathbf{r}_{2, K}^{T} \bar{G}_{\Delta_{K}}^{R}\left(z_{h}\right) \mathbf{r}_{2, K}\right]^{1 / 2} \quad \text { with } \quad z_{h}=u_{h}, v_{h},
$$

collect the anisotropic information associated with $K$, with

$$
\bar{G}_{\Delta_{K}}^{R}(\cdot)=G_{\Delta_{K}}^{R}(\cdot) /\left(|\widehat{K}| \lambda_{1, K} \lambda_{2, K}\right) .
$$


Following Section 4 in [35], we properly merge the two terms in (4.1) to deal with a single metric. This yields

$$
\eta_{K}\left(u_{h}, v_{h}\right)=\mu_{K} \Upsilon_{K}
$$

with

$$
\Upsilon_{K}=\left[s_{K} \mathbf{r}_{1, K}^{T} \Gamma_{K} \mathbf{r}_{1, K}+\frac{1}{s_{K}} \mathbf{r}_{2, K}^{T} \Gamma_{K} \mathbf{r}_{2, K}\right]^{1 / 2},
$$

where the local matrix

$$
\Gamma_{K}=\left[\bar{\rho}_{K}^{A}\left(v_{h}, u_{h}\right)\right]^{2} \bar{G}_{\Delta_{K}}^{R}\left(u_{h}\right)+\left[\bar{\rho}_{K}^{B}\left(v_{h}, u_{h}\right)\right]^{2} \bar{G}_{\Delta_{K}}^{R}\left(v_{h}\right)
$$

merges the anisotropic information provided by $u$ and $v$ suitably weighted via the local residuals. In this way, we are able to grasp all the directional features induced by $u$ and $v$, thus avoiding the metric intersection issue.

Now to minimize the number of mesh elements, we equivalently maximize the area of each element $K$ with the equidistribution constraint, i.e., for each element $K \in \mathcal{T}_{h}^{(j+1)}, \eta_{K}\left(u_{h}, v_{h}\right)=\mu_{K} \Upsilon_{K}=$ TOL $/ \# \mathcal{T}_{h}^{(j)}$, where TOL and $\# \mathcal{T}_{h}^{(j)}$ are the fixed global tolerance and the number of mesh elements in $\mathcal{T}_{h}^{(j)}$, respectively. The maximization is achieved by minimizing the weight $\Upsilon_{K}$ with respect to $s_{K}$ and $\mathbf{r}_{1, K}$, i.e., by solving elementwise the constrained minimization problem

$$
\min _{s_{K} \geq 1, \mathbf{r}_{i, K} \cdot \mathbf{r}_{j, K}=\delta_{i j}} \Upsilon_{K}\left(\mathbf{r}_{1, K}, s_{K}\right),
$$

$\delta_{i j}$ being the Kronecker symbol. Notice that all the quantities involved in (4.3) are computed on the background grid $\mathcal{T}_{h}^{(j)}$. On the other hand, the aspect ratio $s_{K}$ and the unit vectors $\mathbf{r}_{i, K}$ in (4.2) represent our actual unknowns.

According to Proposition 4.2 in [35], we can state the desired minimization result as

Proposition 4.1. Let $\left\{\gamma_{i, K}, g_{i, K}\right\}$ be the eigenvector-eigenvalue pair of $\Gamma_{K}$ with $g_{1, K} \geq g_{2, K}>0$. Then the minimum (4.4) is obtained for the choices

$$
\mathbf{r}_{1, K}=\gamma_{2, K} \quad \text { and } \quad s_{K}=\left(\frac{g_{1, K}}{g_{2, K}}\right)^{1 / 2},
$$

yielding the value $\left(2 \sqrt{g_{1, K} g_{2, K}}\right)^{1 / 2}$ for $\Upsilon_{K}$. Notice that the minimization problem (4.4) is not a computational overhead, since it can be solved analytically via (4.5). Moreover, we observe that the optimal weight $\Upsilon_{K}$ does not depend any more on the aspect ratio.

Finally, the optimal metric $\mathcal{M}^{(j+1)}$ is obtained by exploiting the equidistribution constraint, i.e., by solving the equations

$$
|\widehat{K}|\left(\lambda_{1, K} \lambda_{2, K}\right)^{3 / 2}\left(2 \sqrt{g_{1, K} g_{2, K}}\right)^{1 / 2}=\frac{\text { TOL }}{\# \mathcal{T}_{h}^{(j)}} \quad \text { and } \quad \frac{\lambda_{1, K}}{\lambda_{2, K}}=s_{K}=\left(\frac{g_{1, K}}{g_{2, K}}\right)^{1 / 2} .
$$

System (4.6) provides us with the distinct values

$$
\lambda_{1, K}=\left(\frac{1}{|\widehat{K}| \sqrt{2}}\left(\frac{g_{1, K}}{g_{2, K}^{2}}\right)^{1 / 2} \frac{\mathrm{TOL}}{\# \mathcal{T}_{h}^{(j)}}\right)^{1 / 3}, \quad \lambda_{2, K}=\left(\frac{1}{|\widehat{K}| \sqrt{2}}\left(\frac{g_{2, K}}{g_{1, K}^{2}}\right)^{1 / 2} \frac{\mathrm{TOL}}{\# \mathcal{T}_{h}^{(j)}}\right)^{1 / 3} .
$$

Eventually, the optimal metric $\mathcal{M}^{(j+1)}$ is characterized by $\mathbf{r}_{1, K}$ in (4.5), $\lambda_{1, K}$ and $\lambda_{2, K}$ in (4.7), with $\mathbf{r}_{2, K} \perp \mathbf{r}_{1, K}$. 
4.3. The whole adaptive procedure. Now, we glue the discrete counterpart of Algorithm 1 with the mesh adaptive procedure. In particular, we propose two algorithms, which differ in the way the minimization and the mesh adaptivity are interlaced. For both algorithms, we denote by $\mathcal{T}_{h}$ the mesh used to start up the mesh adaptive procedure.

The first algorithm, which is a variant of ALGORITHM 1 in [10], applies the mesh adaptation after convergence of the minimization algorithm on both $u_{h}$ and $v_{h}$. In particular, after fixing a termination tolerance VTOL $\ll 1$ for the minimization algorithm, a relative tolerance MESHTOL $\ll 1$ on the change of the mesh cardinality, and REFTOL $\ll 1$ which fixes the accuracy on the functional (3.6), the algorithm is the following:

\section{Algorithm 2 (Optimize-then-Adapt).}

1. Set $k=0, j=0, \mathcal{T}_{h}^{(0)}=\mathcal{T}_{h}$;

2. If $k=0$, set $v_{h}^{1}=1$; else $v_{h}^{1}=v_{h}\left(t_{k-1}\right)$;

3. Set $j=0$; errmesh $=1$;

while errmesh $\geq$ MESHTOL do

4. Set $i=1$; $\operatorname{err}=1$;

while err $\geq$ VTOL do

5. $u_{h}^{i}=\arg \min I\left(z_{h}, v_{h}^{i}\right)$; $z_{h} \in X_{h}^{(j)}$

6. $v_{h}^{i+1}=\arg \min I\left(u_{h}^{i}, z_{h}\right)$; $z_{h} \in X_{h}^{(j)}$

7. $\operatorname{err}=\left\|v_{h}^{i+1}-v_{h}^{i}\right\|_{L^{\infty}(\Omega)}$;

8. $i \leftarrow i+1$;

end while

9. Compute the new metric $\mathcal{M}^{(j+1)}$ based on $u_{h}^{i-1}$ and $v_{h}^{i}$ with TOL = REFTOL;

10. Build the adapted mesh $\mathcal{T}_{h}^{(j+1)}$;

11. errmesh $=\left|\# \mathcal{T}_{h}^{(j+1)}-\# \mathcal{T}_{h}^{(j)}\right| / \# \mathcal{T}_{h}^{(j)}$;

12. Set $v_{h}^{1}=\Pi_{j \rightarrow j+1}\left(v_{h}^{i}\right)$;

13. $j \leftarrow j+1$;

end while

14. $u_{h}\left(t_{k}\right)=\Pi_{j-1 \rightarrow j}\left(u_{h}^{i-1}\right) ; v_{h}\left(t_{k}\right)=\Pi_{j-1 \rightarrow j}\left(v_{h}^{i}\right) ; \mathcal{T}_{h}^{k}=\mathcal{T}_{h}^{(j)}$;

15. Set $\mathcal{T}_{h}^{(0)}=\mathcal{T}_{h}^{k}$;

16. $k \leftarrow k+1$;

17. if $k>F$, stop; else goto 2.

The convergence of the mesh adaptivity is checked by monitoring the variation of the number of elements during the adaptivity process. Although this check is not rigorously sound, in practice it provides an effective stopping criterion.

An interpolation step between two successive adapted meshes is also employed before restarting any new optimization or time loop. This is carried out by a suitable interpolation operator, $\Pi_{n \rightarrow n+1}\left(w_{h}\right)$, which maps a finite element function $w_{h}$ defined on $\mathcal{T}_{h}^{n}$ onto the new mesh $\mathcal{T}_{h}^{n+1}$.

This algorithm performs well if the tip of the fracture moves sufficiently slow in time. Indeed, since the coupling between optimization and adaptation is not so tight, a time-adaptivity could be desirable to restrain a fast mesh evolution. Nevertheless, time adaptivity is not able to contain the final evolution steps when the actual fracture lead to a sudden breakdown of the material which splits into two separate parts. This limit can be ascribed also to the deficiency of the employed quasistatic model, which 
clearly fails in describing very fast dynamics.

To dampen the crack propagation, we propose a second algorithm, which introduces a tighter alternation of the optimization and mesh adaptation phases. The meaning of all the involved parameters is the same as in Algorithm 2.

Algorithm 3 (Optimize-and-Adapt).

1. Set $k=0, \mathcal{T}_{h}^{(1)}=\mathcal{T}_{h}$;

2. If $k=0$, set $v_{h}^{1}=1$; else $v_{h}^{1}=v_{h}\left(t_{k-1}\right)$;

3. Set $i=1$; errmesh $=1$; err $=1$;

while errmesh $\geq$ MESHTOL $\&$ err $\geq$ VTOL $\boldsymbol{d o}$

4. $u_{h}^{i}=\arg \min I\left(z_{h}, v_{h}^{i}\right)$; $z_{h} \in X_{h}^{(i)}$

5. $v_{h}^{i+1}=\arg \min I\left(u_{h}^{i}, z_{h}\right)$; $z_{h} \in X_{h}^{(i)}$

6. Compute the new metric $\mathcal{M}^{(i+1)}$ based on $u_{h}^{i}$ and $v_{h}^{i+1}$ with TOL = REFTOL;

7. Build the adapted mesh $\mathcal{T}_{h}^{(i+1)}$;

8. $e r r=\left\|v_{h}^{i+1}-v_{h}^{i}\right\|_{L^{\infty}(\Omega)}$;

9. errmesh $=\left|\# \mathcal{T}_{h}^{(i+1)}-\# \mathcal{T}_{h}^{(i)}\right| / \# \mathcal{T}_{h}^{(i)}$;

10. Set $v_{h}^{1}=\Pi_{i \rightarrow i+1}\left(v_{h}^{i+1}\right)$;

11. $i \leftarrow i+1$;

\section{end while}

12. $u_{h}\left(t_{k}\right)=\Pi_{i-1 \rightarrow i}\left(u_{h}^{i-1}\right) ; v_{h}\left(t_{k}\right)=v_{h}^{1} ; \mathcal{T}_{h}^{k}=\mathcal{T}_{h}^{(i)}$;

13. $\operatorname{Set}_{h}^{(1)}=\mathcal{T}_{h}^{k}$;

14. $k \leftarrow k+1$

15. if $k>F$, stop; else goto 2.

Notice that in this algorithm a single while-loop is involved; the mesh adaptivity is carried out at each iteration, as soon as both $u_{h}$ and $v_{h}$ are available.

5. Numerical experiments. Goal of this section is to assess the robustness of the algorithms proposed in the previous section on some benchmark problems. In particular, to have a comparison solution, we choose the test-cases proposed in [10].
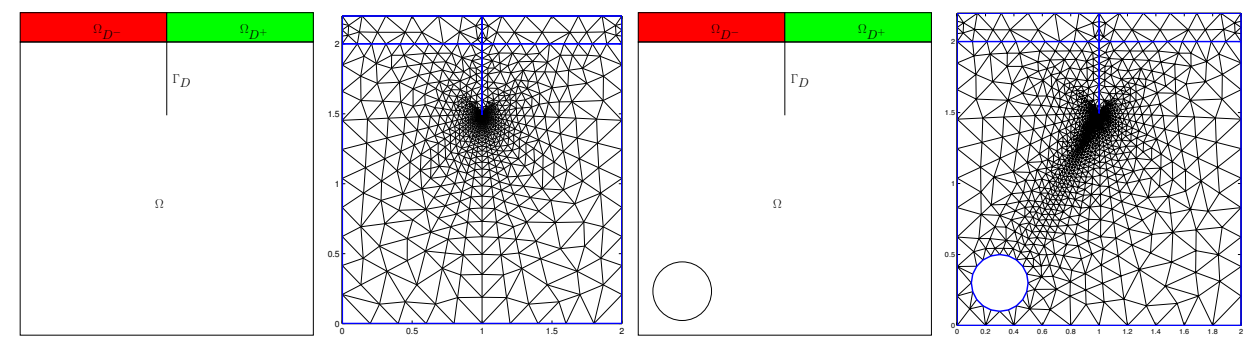

FIG. 5.1. Domain and initial mesh for the straight crack (left pair), and for the curved crack (right pair)

5.1. The straight crack. Let us identify a brittle material of rectangular shape, $\Omega=(0,2) \times(0,2.2)$, containing a slit along $\{1\} \times[1.5,2.2]$ (see Figure 5.1, left), that we approximate with a very thin gap $2 \cdot 10^{-5}$ thick. We apply the antiplane displacement $g(t)=-t$ on $\Omega_{D^{-}}=(0,1) \times(2,2.2), g(t)=t$ on $\Omega_{D^{+}}=(1,2) \times(2,2.2)$. Due to the perfect symmetry of this problem, we expect that the fracture does not bend but that 
it goes straight down starting from the tip of the slit.

As an initial grid, we pick the uniform unstructured mesh in Figure 5.1, left. We consider a time window $[0,1.5]$ sufficiently wide to contain the whole phenomenon. Concerning the parameters involved in both the algorithms, we choose the ones in Table 5.1.

TABLE 5.1

The straight crack: parameters involved in Algorithms 2 and 3

\begin{tabular}{|c|c|c|c|}
\hline$\varepsilon=2 \cdot 10^{-2}$ & $\eta=10^{-5}$ & $\gamma_{A}=\gamma_{B}=10^{-5}$ & $\Delta t=10^{-2}$ \\
\hline $\mathrm{CRTOL}=3 \cdot 10^{-4}$ & $\mathrm{VTOL}=2 \cdot 10^{-3}$ & $\mathrm{MESHTOL}=10^{-2}$ & $\mathrm{REFTOL}=10^{-2}$ \\
\hline
\end{tabular}

Figure 5.2 compares the crack path yielded by the two algorithms. Notice that the final part of the crack delivered by Algorithm 3 is slightly straighter and more regular. This is likely due to the fact that Algorithm 2 is more sensitive to the possible coarseness of the mesh ahead of the tip. As a consequence, when the crack reaches the final stage, it tries to enter a region where the mesh has not been modified yet. Conversely, the tighter interplay between optimization and mesh adaptation in Algorihm 3 lets the crack find an already properly adapted mesh. An additional difference is the time when the breakdown is detected, i.e., $t=1.36$ for Algorihm 2 and $t=1.33$ in the case of Algorihm 3, compared with $t=1.24$ in [10]. Indeed, since in the first algorithm we do not update the mesh during the minimization process, it can happen that the crack growing is slowed down in order to find a good compromise between the actual mesh and the fracture evolution. We additionally observe that for both Algorithms 2 and 3, the time of initiation of the fracture actually occurs later, i.e., at time $t=0.35$, than the experiments in [10], where $t=0.25$. We ascribe this discrepancy to the finite-width representation of the initial crack path via the vertical slit, while in [10] this is modeled via an actual 1-dimensional manifold. Concerning the computational effort, the run time of Algorithms 2 and 3 is $1541.30 \mathrm{~s}$ and $1639.29 \mathrm{~s}$, respectively.
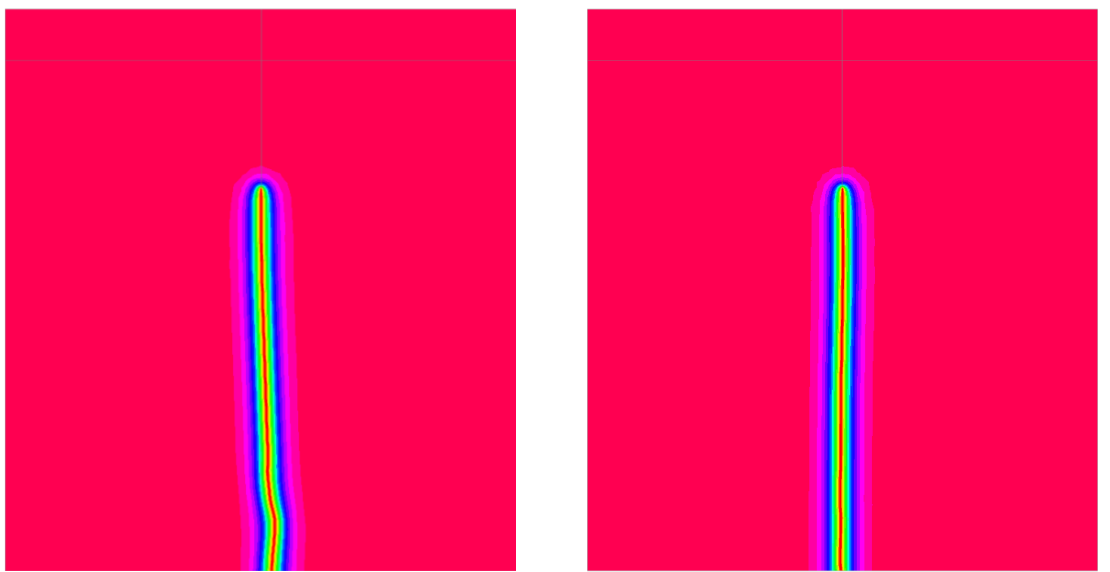

FIG. 5.2. The straight crack: v-field at the final time yielded by Algorihm 2 (left) and Algorihm 3 (right)

Figure 5.3 shows the adatpted mesh $\mathcal{T}_{h}^{\text {algo2 }}$ and $\mathcal{T}_{h}^{\text {algo } 3}$ obtained by the two algorithms at the final time. The meshes, consisting of 38299 and 33927 elements respec- 

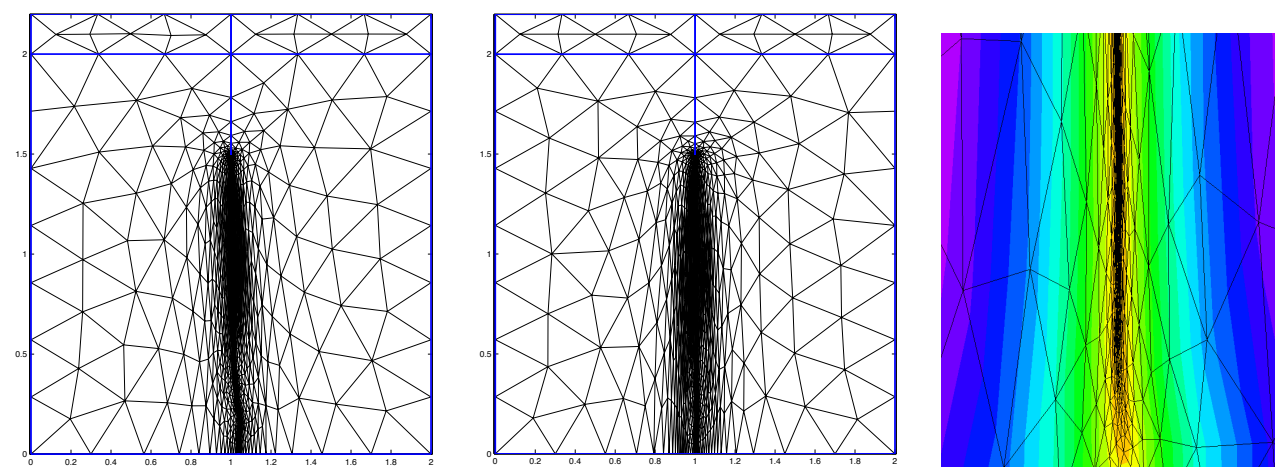

FIG. 5.3. The straight crack: final anisotropic adapted mesh provided by Algorithm 2 (left); final anisotropic adapted mesh (center) and zoom in (right) delivered by Algorithm 3

tively, exhibit really stretched elements which closely follow the crack path, whereas the mesh is very coarse in the unfractured domain, i.e., where $v_{h} \simeq 1$. The maximum aspect ratio is $s_{K}=2154.3$ for $\mathcal{T}_{h}^{\text {algo } 2}$ and $s_{K}=1891.5$ for $\mathcal{T}_{h}^{\text {algo } 3}$. The close up in Figure 5.3 at time $t=1.21$ highlights the strongly anisotropy of the mesh far from the crack tip. We observe instead that the triangles closer to the tip are still rather isotropic. This should guarantee that the next advancing step of the crack is not biased by the directionality of the elements. After $[8,14]$, there has been the perception that anisotropic mesh adaptation may influence the propagation of the fracture, in particular its initiation [13]. However, it seems that the numerical procedure that we propose is in practice robust and stable thanks to its automatic capability of yielding a rounded tip.
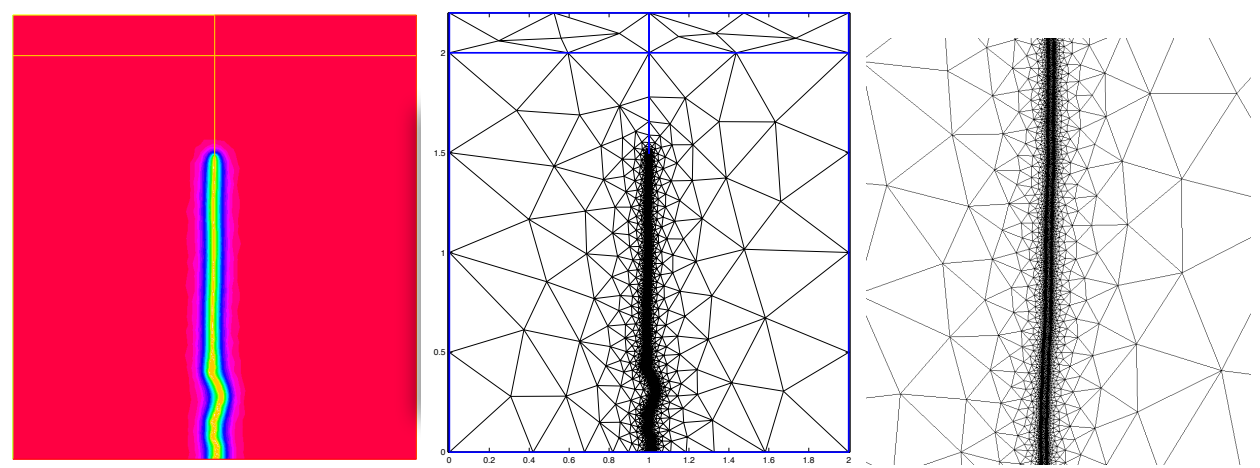

FIG. 5.4. The straight crack: v-field (left), final adapted mesh (center), and zoom in (right) at the final time in the case of the isotropic counterpart of Algorithm 2.

Figure 5.4 provides the $v$-field and the final adapted mesh for an isotropic adaptation, obtained by enforcing $s_{K}=1$ for all $K \in \mathcal{T}_{h}$ in Algorithm 2. The crack is detected also in this case even though the required number of elements is far larger, i.e., 78025 triangles versus 38299. Moreover, a slightly wavier path is exhibited with respect to Figure 5.2, left.

5.2. The curved crack. This second test case is meant to assess whether the fracture changes direction if the domain exhibits a weak inset, such as a hole. The 
computational domain is the same as in the previous test case with the additional presence of a circular hole of radius 0.2, centered at $(0.3,0.3)$ (see Figure 5.1, right). The presence of the hole introduces an element of weakness in the material. As a consequence, due to energy arguments, we expect that the fracture bends its path towards the hole instead of proceedings along a straight line. As observed in [10], this test case is more challenging than the previous one. Therefore, we choose a tighter tolerance, i.e., REFTOL $=10^{-3}$. The simulated crack path is very stable with respect to the choice of the parameters, as long as they are not larger than those in Table 5.1 (see [3], where an extensive sensitivity analysis to the parameter tuning is carried out).
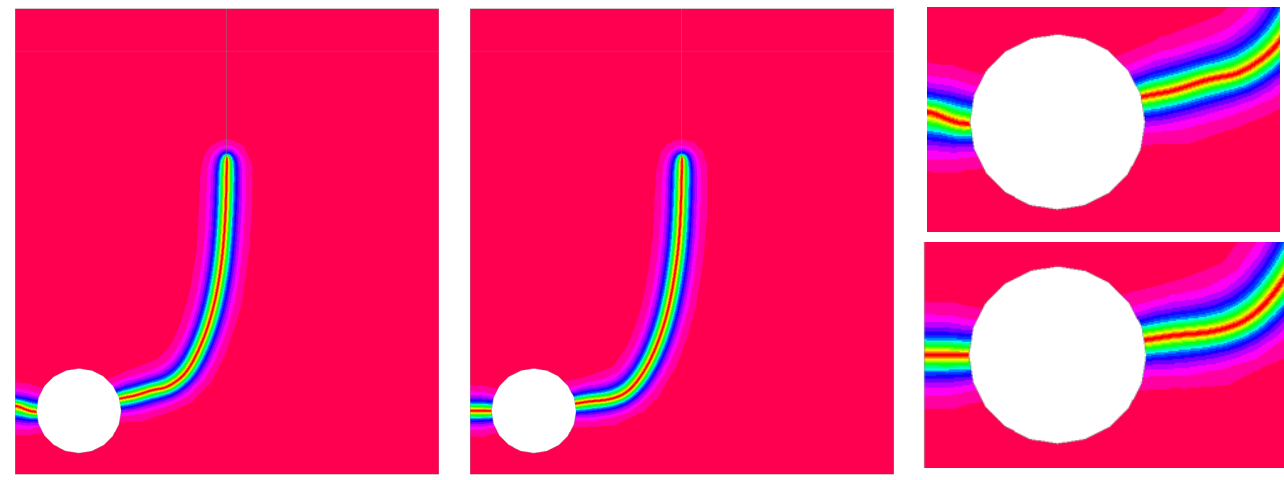

FIG. 5.5. The curved crack: $v$-field at the final time provided by Algorithm 2 (left) and by Algorithm 3 (center); zooms in around the hole for Algorithm 2 (top-right) and for Algorithm 3 (bottom-right)

In Figure 5.5, we show the $v$-field at the final time yielded by the two algorithms. In both cases, the crack enters the hole. As already observed in the previous test case, Algorithm 2 leads a more "bumpy" crack path ahead of the hole (compare Figure 5.5, left with Figure 5.5, center, and the corresponding zooms in).
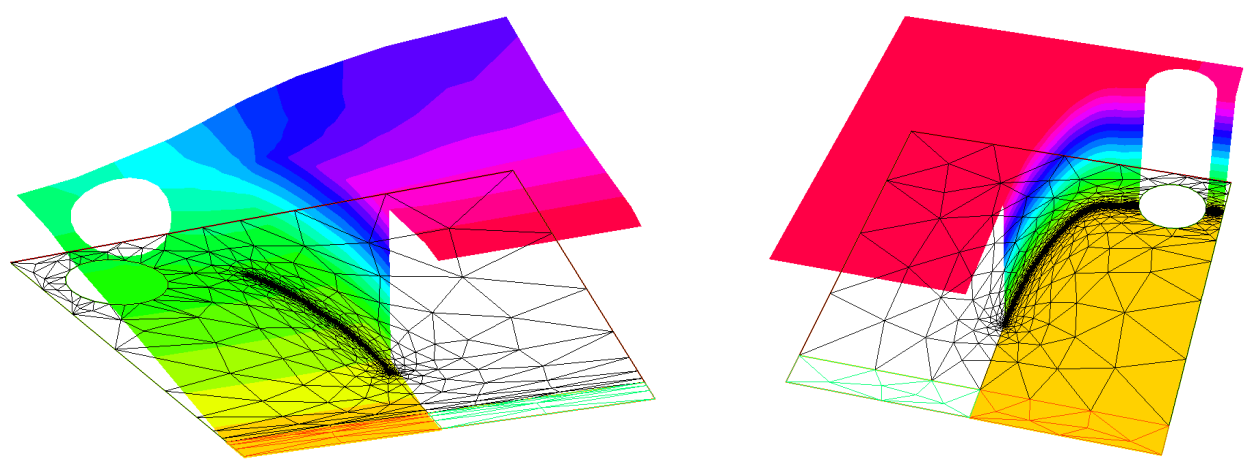

FIG. 5.6. The curved crack: $u$-field and adapted mesh at $t=1.37$ (left) and at $t=1.43$ (right) provided by Algorithm 3

Figure 5.6 displays the $u$-field superposed to the adapted meshes at $t=1.37$ (left) and at $t=1.43$ (right) in the case of Algorithm 3. A very steep ridge is evident where the tearing apart is exerted. The mesh in both cases follows very closely the 

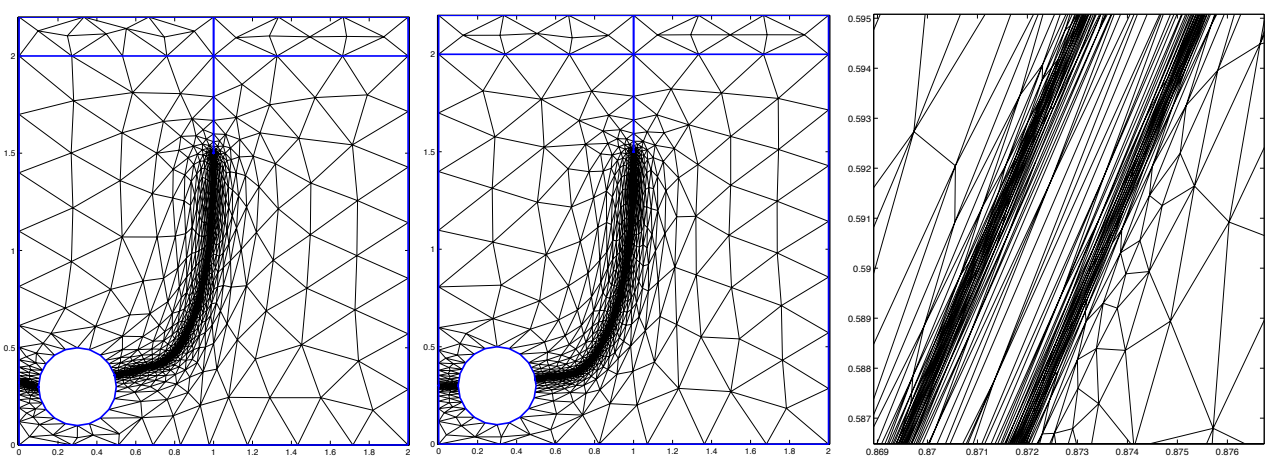

FIG. 5.7. The curved crack: final anisotropic adapted mesh provided by Algorithm 2 (left); final anisotropic adapted mesh (center) and zoom in (right) delivered by Algorithm 3

crack propagation. A top view of the final adapted meshes generated via Algorithm 2 and 3 is provided in Figure 5.7, together with a detail of the second mesh. Notice that the anisotropic adaptive procedure is able to detect the presence of a very fine structure inside the crack in correspondence with the ridges. Moreover, the cardinality of the two meshes is very different: Algorithm 2 employs 48599 elements in contrast to Algorithm 3 which demands only 15987 triangles. The maximum aspect ratio is $s_{K}=1525.3$ for $\mathcal{T}_{h}^{\text {algo } 2}$ and $s_{K}=1469.9$ for $\mathcal{T}_{h}^{\text {algo } 3}$.
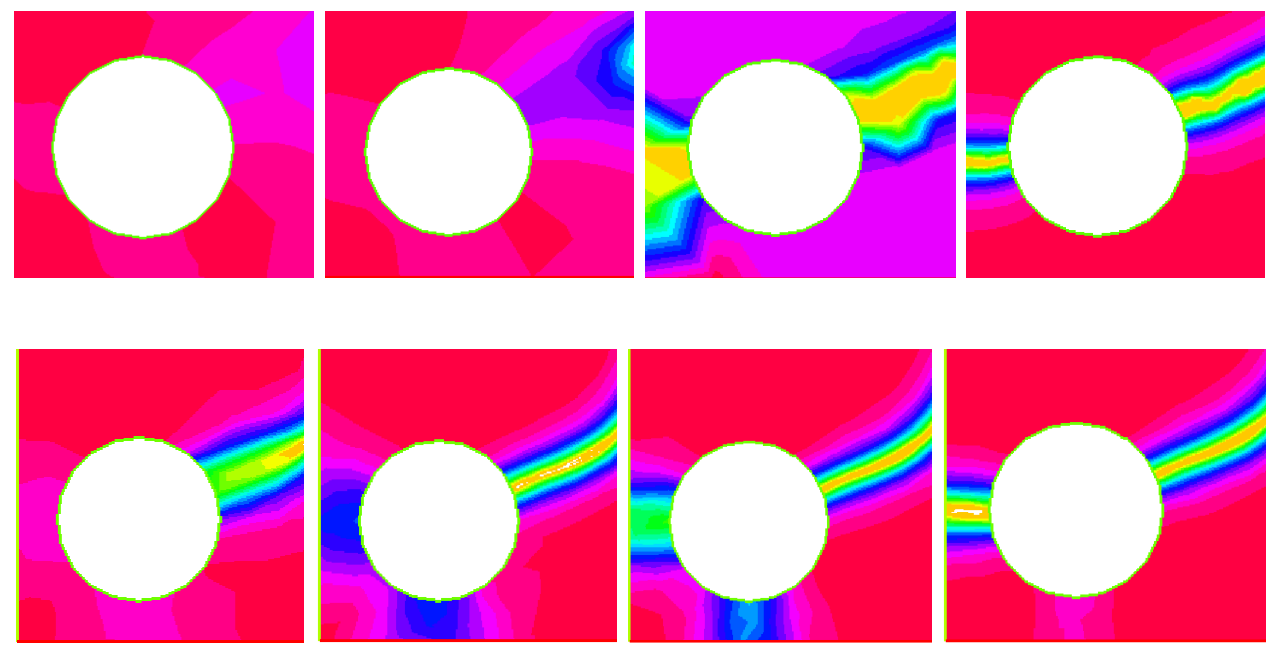

FIG. 5.8. The curved crack: successive iterations of Algorithm 2 (top) and Algorithm 3 (bottom) in the breakdown phase

Figure 5.8 shows four snapshots close to the breakdown time by comparing four successive iterations of Algorithm 2 (top) with Algorithm 3 (bottom). In the case of Algorithm 2, the crack, after entering the hole, reaches a region where the mesh is still coarse. Afterwards, the grid is correctly refined. On the contrary, the strategy optimize-and-adapt detects a sharper path already on entering the hole. Moreover, before converging to the failure of the material, two possible paths, energetically equivalent, pop out past the hole.

Figure 5.9, left provides the time evolution of the energy, $E_{e}+E_{f}$ in (1.2). The 

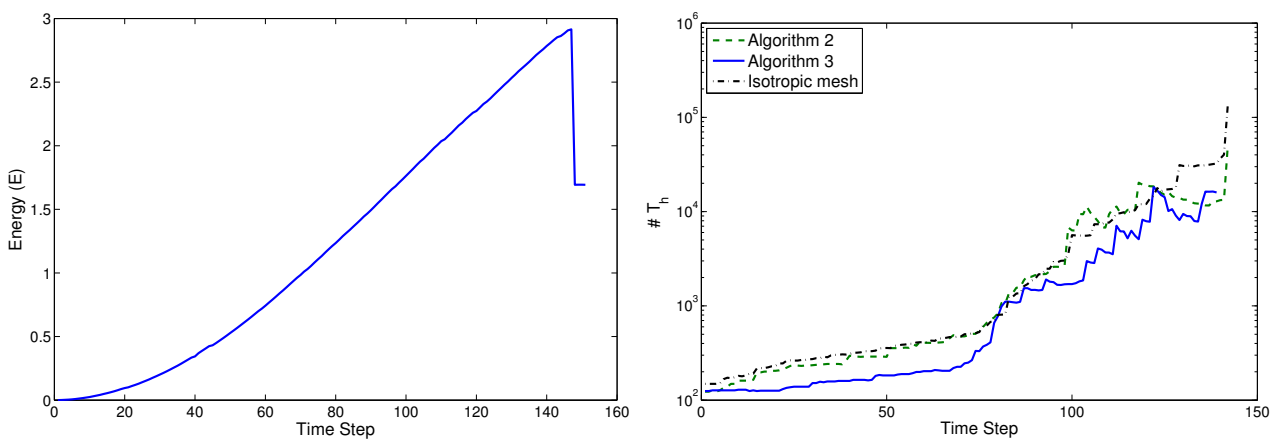

FIG. 5.9. The curved crack: energy (left) and cardinality (right) evolution for the anisotropic meshes yielded by the two Algorithms and for the isotropic counterpart of Algorithm 3

energy constantly increases. During the very first phase, when the crack has not started yet, the dominant contribution to the energy is $E_{e}$. Successively, after the onset of the crack propagation at $t=0.35$, the fictitious energy $E_{f}$ contributes to the whole energy. After the breakdown of the material $(t=1.43)$, the energy decreases suddenly since the elastic energy abruptly vanishes. On physical grounds, we would expect the energy to go to zero after the breakdown. However, the fictitious energy leaves a trace which never disappears. In Figure 5.9, right we compare the trend of the cardinality of the anisotropic meshes associated with the two adaptive algorithms along with the isotropic counterpart of Algorithm 3. It is evident the saving brought by Algorithm 3 and the strong increase exhibited by Algorithm 2 in the very final phase.

Moreover, the isotropic variant of Algorithm 3 generates a larger number of elements throughout all the time evolution. In particular, at the final time the isotropic mesh consists of 131367 triangles, i.e., about a factor 8 with respect to the best performing Algorithm 3.
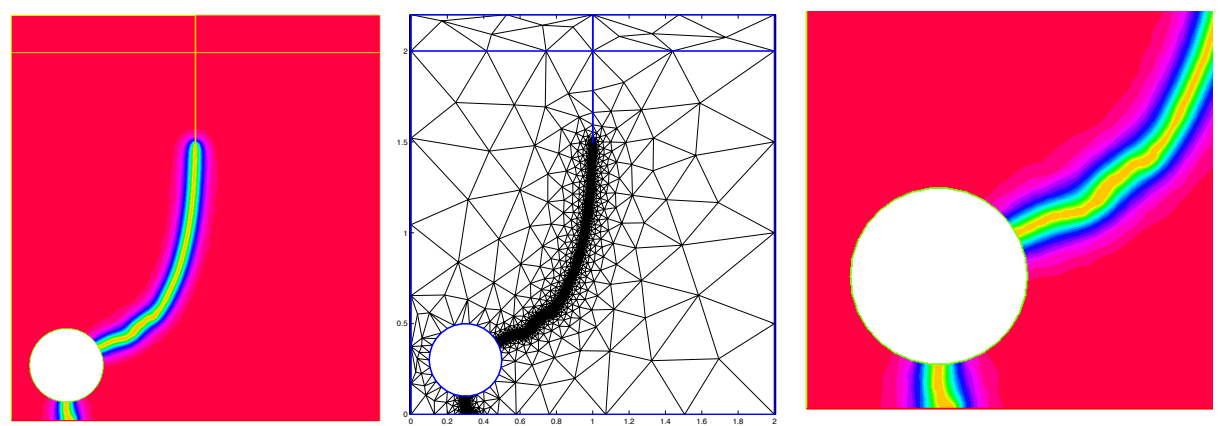

FIG. 5.10. The curved crack: v-field (left) at the final time, final adapted mesh (center) and zoom in (right) on the hole in the case of the isotropic counterpart of Algorithm 3

Finally, in Figure 5.10, we collect the results obtained through Algorithm 3 when we enforce an isotropic mesh adaptation, i.e., $s_{K}=1$ for all $K \in \mathcal{T}_{h}$. We first recognize the different path undertaken by the crack, namely, the crack leaves the hole on the bottom instead on the left. However, this different path could be plausible from a physical point of view since both the paths are energetically equivalent. On the other hand, physical experiments select the one in Figure 5.5 as the most likely ([32, 38], 
see also their related works $[9,39])$. The alternative path in Figure 5.10 suggests that a more thorough numerical investigation should be carried out in order to properly calibrate the algorithm parameters for isotropic meshes.

Future developments of the proposed approach concern the modeling of fractures under plane-strain elasticity as well as more general mathematical models such as the ones introduced in [12].

Acknowledgments. Marco Artina and Massimo Fornasier acknowledge the financial support of the International Research Training Group IGDK 1754 "Optimization and Numerical Analysis for Partial Differential Equation with Nonsmooth Structures" of the German Science Foundation.

Marco Artina thanks the MOX - Modeling and Scientific Computing Laboratory at Politecnico di Milano for the hospitality during part of the preparation of this work.

Simona Perotto acknowledge the financial support of the Project MIUR "Innovative methods for water resources under hydro-climatic uncertainty scenarios", PRIN $2010 / 2011$.

\section{REFERENCES}

[1] L. Ambrosio and V. M. Tortorelli. Approximation of functional depending on jumps by elliptic functional via $\gamma$-convergence. Comm. Pure Appl. Math., 43(8):999-1036, 1990.

[2] L. Ambrosio and V. M. Tortorelli. On the approximation of free discontinuity problems. Boll. Unione Mat. Ital., 6(1):105-123, 1992.

[3] M. Artina, M. Fornasier, S. Micheletti, and S. Perotto. Anisotropic adaptive meshes for brittle fractures: parameter sensitivity. MOX-report, 58/2013.

[4] M. Artina, M. Fornasier, and F. Solombrino. Linearly constrained nonsmooth and nonconvex minimization. SIAM J. Optim., 23(3):1904-1937, 2013.

[5] G. Bellettini and A. Coscia. Discrete approximation of a free discontinuity problem. Numer. Funct. Anal. Optim., 15(3-4):201-224, 1994.

[6] B. Bourdin. Numerical implementation of the variational formulation for quasi-static brittle fracture. Interfaces Free Bound., 9(3):411-430, 2007.

[7] B. Bourdin. The variational formulation of brittle fracture: numerical implementation and extensions. In IUTAM Symposium on Discretization Methods for Evolving Discontinuities, pages 381-393. Springer, New York, 2007.

[8] B. Bourdin and A. Chambolle. Implementation of an adaptive finite-element approximation of the Mumford-Shah functional. Numer. Math., 85(4):609-646, 2000.

[9] B. Bourdin, G. Francfort, and J.-J. Marigo. Numerical experiments in revisited brittle fracture. J. Mech. Phys. Solids, 48(4):797 - 826, 2000.

[10] S. Burke, C. Ortner, and E. Süli. An adaptive finite element approximation of a variational model of brittle fracture. SIAM J. Numer. Anal., 48(3):980-1012, 2010.

[11] S. Burke, C. Ortner, and E. Süli. Adaptive finite element approximation of the Francfort-Marigo model of brittle fracture. In Approximation and Computation, volume 42 of Springer Optim. Appl., pages 297-310. Springer, New York, 2011.

[12] S. Burke, C. Ortner, and E. Süli. An adaptive finite element approximation of a generalized Ambrosio-Tortorelli functional. Math. Models Methods Appl. Sci., 23(9):1663-1697, 2013.

[13] A. Chambolle. Personal communication.

[14] A. Chambolle and G. Dal Maso. Discrete approximation of the Mumford-Shah functional in dimension two. M2AN Math. Model. Numer. Anal., 33(4):651-672, 1999.

[15] Ph. G. Ciarlet. The Finite Element Method for Elliptic Problems. North-Holland, Amsterdam, 1978.

[16] Ph. G. Ciarlet and P.-A. Raviart. Maximum principle and uniform convergence for the finite element method. Comput. Methods Appl. Mech. Engrg., 2(1):17-31, 1973.

[17] $\mathrm{Ph}$. Clément. Approximation by finite element functions using local regularization. RAIRO Anal. Numér., 2:77-84, (1975).

[18] G. Dal Maso. An introduction to $\Gamma$-convergence. Birkhäuser, Basel, 1993.

[19] G. Dal Maso and R. Toader. A model for the quasi-static growth of brittle fractures based on local minimization. Math. Models Methods Appl. Sci., 12(12):1773-1799, 2002. 
[20] G. Dal Maso and R. Toader. A model for the quasi-static growth of brittle fractures: existence and approximation results. Arch. Ration. Mech. Anal., 162(2):101-135, 2002.

[21] L. Dedè, S. Micheletti, and S. Perotto. Anisotropic error control for environmental applications. Appl. Numer. Math., 58(9):1320-1339, 2008.

[22] M. Focardi. On the variational approximation of free-discontinuity problems in the vectorial case. Math. Models Methods Appl. Sci., 11(04):663-684, 2001.

[23] L. Formaggia and S. Perotto. New anisotropic a priori error estimates. Numer. Math., 89(4):641-667, 2001.

[24] L. Formaggia and S. Perotto. Anisotropic error estimates for elliptic problems. Numer. Math., 94:67-92, 2003.

[25] G. Francfort and J.-J. Marigo. Revisiting brittle fracture as an energy minimization problem. J. Mech. Phys. Solids, 46(8):1319-1342, 1998.

[26] G. A. Francfort and C. J. Larsen. Existence and convergence for quasi-static evolution in brittle fracture. Comm. Pure Appl. Math., 56(10):1465-1500, 2003.

[27] P.-L. George and H. Borouchaki. Delaunay Triangulation and Meshing. Application to Finite Elements. Edition Hermès, Paris, 1998.

[28] A. Giacomini. Ambrosio-Tortorelli approximation of quasi-static evolution of brittle fractures. Calc. Var. Partial Differential Equations, 22(2):129-172, 2005.

[29] A. A. Griffith. The phenomena of rupture and flow in solids. Phil. Trans. R. Soc. Lond. Series A, 221:163-198, 1921.

[30] F. Hecht. New developement in Freefem++. J. Numer. Math., 20(3-4):251-265, 2012.

[31] S. Korotov, M. Kř́žzek, and P. Neittaanmäki. Weakened acute type condition for tetrahedral triangulations and the discrete maximum principle. Math. Comp., 70(233):107-119, 2001.

[32] J.-J. Marigo. Personal communication.

[33] S. Micheletti and S. Perotto. Reliability and efficiency of an anisotropic Zienkiewicz-Zhu error estimator. Comput. Methods Appl. Mech. Engrg., 195(9):799-835, 2006.

[34] S. Micheletti and S. Perotto. Output functional control for nonlinear equations driven by anisotropic mesh adaption: the Navier-Stokes equations. SIAM J. Sci. Comput., 30(6):2817-2854, 2008.

[35] S. Micheletti and S. Perotto. The effect of anisotropic mesh adaptation on PDE-constrained optimal control problems. SIAM J. Control. Optim., 49(4):1793-1828, 2011.

[36] S. Micheletti, S. Perotto, and M. Picasso. Stabilized finite elements on anisotropic meshes: a priori error estimates for the advection-diffusion and the Stokes problems. SIAM J. Numer. Anal., 41(3):1131-1162, 2003.

[37] D. Mumford and J. Shah. Optimal approximations by piecewise smooth functions and associated variational problems. Comm. Pure Appl. Math., 42(5):577-685, 1989.

[38] M. Negri. Personal communication.

[39] M. Negri. A finite element approximation of the Griffith's model in fracture mechanics. Numer. Math., 95(4):653-687, 2003.

[40] G. Strang and G. J. Fix. An Analysis of the Finite Element Method, volume 212. Prentice-Hall Englewood Cliffs, NJ, 1973.

[41] V. Thomée. Galerkin Finite Element Methods for Parabolic Problems, volume 25. Springer Verlag, Berlin, 1997.

[42] O. C. Zienkiewicz and J. Z. Zhu. The superconvergent patch recovery and a posteriori error estimates. part 1: The recovery technique. Int. J. Numer. Methods Engrg., 33(7):13311364, 1992. 